\title{
A FREE BOUNDARY VALUE PROBLEM FOR THE FULL EULER SYSTEM AND 2-D TRANSONIC SHOCK IN A LARGE VARIABLE NOZZLE
}

\author{
Jun Li, Zhouping Xin, AND Huicheng Yin
}

\begin{abstract}
In this paper, we establish the existence and uniqueness of a transonic shock solution to the full steady compressible Euler system in a class of de Laval nozzles with a large straight divergent part when a given variable exit pressure lies in a suitable range. Thus, for this class of nozzles, we have solved the transonic shock problem posed by Courant-Friedrichs in Section 147 of [5]. By introducing a new elaborate iteration scheme, we are able to solve this boundary value problem for a coupled elliptic-hyperbolic system with a free boundary without some stringent requirements in the previous studies. One of the key ingredients in this approach is to solve a boundary value problem for a first order linear system with nonlocal terms and a free parameter.
\end{abstract}

\section{Introduction and main results}

In this paper, we focus on the existence and uniqueness of a transonic shock solution in a de Laval nozzle with a large straight diverging part for the two dimensional full steady compressible Euler system. This is motivated by the following well-known transonic shock phenomena described by Courant-Friedrichs in Section 147 of [5]: Given the appropriately large exit pressure $p_{e}(x)$, if the upstream flow is still supersonic behind the throat of the nozzle, then at a certain place in the diverging part of the nozzle a shock front intervenes and the gas is compressed and slowed down to subsonic speed so that the position and the strength of the shock front are automatically adjusted such that the end pressure becomes $p_{e}(x)$.

The 2-D full steady Euler system is

$$
\left\{\begin{array}{l}
\operatorname{div}(\rho u)=0, \\
\operatorname{div}(\rho u \otimes u)+\nabla P=0, \\
\operatorname{div}\left(\rho u\left(e+\frac{1}{2}|u|^{2}\right)+u P\right)=0,
\end{array}\right.
$$

where $u=\left(u_{1}, u_{2}\right)$ is the velocity, $\rho$ is the density, $P$ is the pressure, $e$ is the internal energy and $S$ is the special entropy respectively. Moreover, for ideal polytropic gases, the equations of states are given by $P=A \rho^{\gamma} e^{\frac{S}{c_{v}}}$ and $e=\frac{P}{(\gamma-1) \rho}$, here $A, c_{v}$ and $\gamma$ $(1<\gamma<3)$ are positive constants.

Assume that the nozzle walls $\Gamma_{1}$ and $\Gamma_{2}$ are $C^{3, \alpha}-$ regular for $X_{0}-1<r=$ $\sqrt{x_{1}^{2}+x_{2}^{2}}<X_{0}+1$ (here $0<\alpha<1$, and $X_{0}>1$ is a fixed constant) and $\Gamma_{i}$

Received by the editors February 16, 2009.

2000 Mathematics Subject Classification. 35L70, 35L65, 35L67, 76N15.

Key words and phrases. Steady Euler system, supersonic flow, subsonic flow, transonic shock, large variable nozzle. 
consists of two curves $\Gamma_{i}^{1}$ and $\Gamma_{i}^{2}$ with $\Gamma_{1}^{1}$ and $\Gamma_{2}^{1}$ including the walls for the converging part of the nozzle, while $\Gamma_{1}^{2}$ and $\Gamma_{2}^{2}$ being the divergent part, which are represented by $x_{2}=(-1)^{i} x_{1} \tan \theta_{0}$ with $x_{1}>0$ and $0<\theta_{0}<\frac{\pi}{2}$. Furthermore, without loss of generality and for simplicity, it is assumed that the supersonic incoming flow $\left(u_{1,0}^{-}(x), u_{2,0}^{-}(x), P_{0}^{-}(x), S_{0}^{-}(x)\right)$ is $C^{\infty}$ - smooth and symmetric near $r=X_{0}$ so that $u_{i, 0}^{-}(x)=\frac{U_{0}^{-}(r) x_{i}}{r}(i=1,2), P_{0}^{-}(x)=P_{0}^{-}(r)$ and $S_{0}^{-}(x)=S_{0}^{-}$( $S_{0}^{-}$is a constant $)$. It is noted that such an assumption can be easily realized by the hyperbolicity of the supersonic flow and the symmetric property of the nozzle walls for $X_{0}<r<X_{0}+1$, one can see $[8]$.

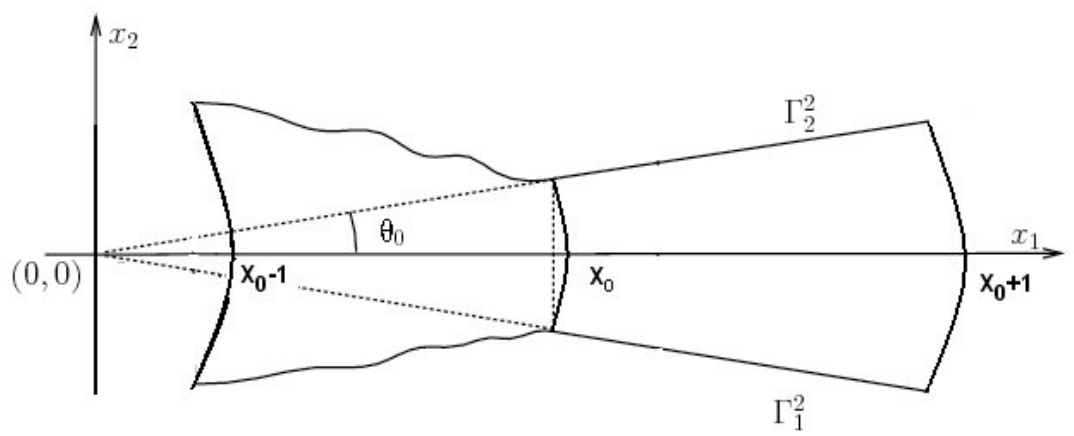

Suppose that the transonic shock curve $\Sigma$ and the flow behind $\Sigma$ are denoted by $x_{1}=\eta\left(x_{2}\right)$ and $\left(u^{+}, P^{+}, S^{+}\right)(x)$ respectively. Then it follows from the R-H conditions on $\Sigma$ that

$$
\left\{\begin{array}{l}
{\left[\rho u_{1}\right]-\eta^{\prime}\left(x_{2}\right)\left[\rho u_{2}\right]=0,} \\
{[\rho u \otimes u+P I] \cdot\left(1,-\eta^{\prime}\left(x_{2}\right)\right)=0} \\
{\left[\left(\rho e+\frac{1}{2} \rho|u|^{2}+P\right) u_{1}\right]-\eta^{\prime}\left(x_{2}\right)\left[\left(\rho e+\frac{1}{2} \rho|u|^{2}+P\right) u_{2}\right]=0}
\end{array}\right.
$$

where $I$ is the $2 \times 2$ identity matrix.

In addition, $P(x)$ satisfies the physical entropy condition (see [5]):

$$
P^{+}(x)>P^{-}(x) \quad \text { on } \quad x_{1}=\eta\left(x_{2}\right) .
$$

On the exit of the nozzle, the end pressure is prescribed by

$$
P^{+}(x)=P_{e}+\varepsilon P_{0}(\theta) \quad \text { on } \quad r=X_{0}+1,
$$

here $\varepsilon>0$ is suitable small, $\theta=\arctan \frac{x_{2}}{x_{1}}, P_{0}(\theta) \in C^{2, \alpha}\left(\left[-\theta_{0}, \theta_{0}\right]\right)$ with $P_{0}^{\prime}\left( \pm \theta_{0}\right)=0$, the constant $P_{e}$ denotes by the end pressure for which a symmetric transonic shock lies at the position $r=r_{0}$ with $r_{0} \in\left(X_{0}, X_{0}+1\right)$ and the supersonic incoming flow is given by $\left(U_{0}^{-}(r), P_{0}^{-}(r), S_{0}^{-}\right)$in the domain $\left\{r: X_{0} \leq r \leq X_{0}+1\right\}$. For more details, one can see Section 147 of [5] or Theorem 1.1 of [16].

Since the flow is tangent to the nozzle walls $x_{2}=(-1)^{i} x_{1} \tan \theta_{0}(i=1,2)$, then

$$
u_{2}^{+}=(-1)^{i} u_{1}^{+} \tan \theta_{0} \quad \text { on } \quad x_{2}=(-1)^{i} x_{1} \tan \theta_{0} .
$$

As been stated in Section 147 of [5] (see also Theorem 1.1 of [16]), under the above assumptions on the nozzle and the symmetric supersonic flow near the throat 
of the nozzle, there exists a unique symmetric transonic shock solution with a shock position $r=r_{0}$ for the given constant end pressure $P_{e}$. This solution will be called the background solution in this paper. Let $\left(U_{0}^{+}(r), P_{0}^{+}(r), S_{0}^{+}\right)\left(S_{0}^{+}\right.$is a constant) be the subsonic part of the background solution for $r_{0}<r<X_{0}+1$, which can be extended into the domain $\left\{r: r_{0}-\delta_{0} \leq r \leq X_{0}+1\right\} \quad\left(\delta_{0}>0\right.$ is some constant depending only on the supersonic incoming flow) (see Theorem 1.1 of [16]). The corresponding extension will be denoted by $\left(\hat{U}_{0}^{+}(r), \hat{P}_{0}^{+}(r), S_{0}^{+}\right)$.

The main result in this paper is

Theorem 1.1. (Existence and Uniqueness)

Under the assumptions on the nozzle walls and supersonic incoming flow, there exists a constant $\varepsilon_{0}>0$ such that for all $\varepsilon \in\left(0, \varepsilon_{0}\right]$, the problem (1.1)-(1.5) has a unique transonic shock solution $\left(u^{+}, P^{+}, S^{+} ; \eta\left(x_{2}\right)\right)$ which admits the following properties:

(i). $\eta\left(x_{2}\right) \in C^{3, \alpha}\left[x_{2}^{1}, x_{2}^{2}\right]$, and

$$
\left\|\eta\left(x_{2}\right)-\sqrt{r_{0}^{2}-x_{2}^{2}}\right\|_{C^{3, \alpha}\left[x_{2}^{1}, x_{2}^{2}\right]} \leq C_{0} \varepsilon
$$

where $\left(x_{1}^{i}, x_{2}^{i}\right)$ stands for the intersection points of $x_{1}=\eta\left(x_{2}\right)$ with $x_{2}=(-1)^{i} x_{1} \tan \theta_{0}$ for $i=1,2$, and the positive generic constant $C_{0}$ depends only on $\alpha$ and the supersonic incoming flow.

(ii). $\left(u^{+}, P^{+}, S^{+}\right)(x) \in C^{2, \alpha}\left(\bar{\Omega}_{+}\right)$, and

$$
\left\|\left(u^{+}, P^{+}, S^{+}\right)-\left(\hat{u}_{0}^{+}, \hat{P}_{0}^{+}, S_{0}^{+}\right)\right\|_{C^{2, \alpha}\left(\bar{\Omega}_{+}\right)} \leq C_{0} \varepsilon,
$$

where $\Omega_{+}$is the subsonic region given by

$$
\Omega_{+}=\left\{\left(x_{1}, x_{2}\right): \eta\left(x_{2}\right)<x_{1}<\sqrt{\left(X_{0}+1\right)^{2}-x_{2}^{2}},\left|x_{2}\right|<x_{1} \tan \theta_{0}\right\},
$$

and $\left(\hat{u}_{0}^{+}, \hat{P}_{0}^{+}\right)=\left(\hat{U}_{0}^{+}(r) \frac{x}{r}, \hat{P}_{0}^{+}(r)\right)$.

Remark 1.1. It should be noted that the previous known results on the uniqueness and/or existence of transonic shocks in a nozzle in [3]-[4], [6], [12]-[13] and [15]-[16] require that the nozzle walls are changing slowly, and either the transonic shock curve goes through a fixed point a priorily with some modified artificial boundary conditions or solutions with special symmetries.

Remark 1.2. In Theorem 1.1, in contrast to the existence and uniqueness results of [9], there is no requirement that the diverging part of the nozzle changes slowly.

There have been many works on general transonic shock problems or general transonic flow problems (see [2]-[6], [9], [12],[13],[14],[15],[16] and the references therein). We will mention only some of the recent results concerning the transonic shocks of steady Euler system in a finite nozzle. For the full steady Euler system in slowly varying nozzle, the uniqueness and existence of transonic shock wave patterns have been investigated under the key additional assumption that the transonic shock goes through a fixed point with various boundary conditions [3]-[4], [15]-[16]. However, due to the additional assumption that the shock goes through a fixed point and/or rather different boundary conditions at the exit [3]-[4], [15]-[16], all those results cannot be applied to the transonic shock problem proposed by Courant-Friedrichs in [5]. Until recently, the first positive result confirming the transonic shock flow pattern has been established by the authors in [9] for a class of 2-D slowly-varying finite nozzles without additional artificial assumptions. In this paper, we proposed a new iteration scheme 
to solve the transonic shock problem for the full steady Euler system without the key assumption that the nozzle is slowly varying.

We now comment on the proofs of the main results in this paper. The transonic shock problem in a nozzle proposed by Courant-Friedrichs can be reduced to a boundary value problem for the full steady Euler system in the subsonic domain with a free boundary (the transonic shock curve or surface). It is noted that for a slowly varying finite nozzle with some suitable boundary conditions on the exit other than the fixed exit pressure, one can directly construct a contractible mapping to establish the existence and uniqueness of a transonic shock solution in a nozzle under the key assumption that the shock surface goes through a fixed point in advance. However, such an approach cannot be applied to the transonic shock problem posed by Courant-Friedrichs with the fixed given exit pressure, since it has been shown that for a class of flat or de Laval nozzles, this problem has no solution if the shock surface is assumed to go through a fixed point in advance [15]. Thus the shock position must be completely free and should be determined as part of the solution depending on the supersonic incoming flow, the geometry of the nozzle, and the exit pressure as in the quasi-one dimensional case [5], and some new ideas other than those used in [3]-[4], [9], [15]-[16] are needed. In [9], for the class of nozzles considered in this paper with the additional assumptions that the diverging part of the nozzle varies slowly (i.e. $\theta_{0}$ is suitably small), the authors were able to establish a priorily the important property that the shock position on the wall of the nozzle depends monotonically and continuously on the given exit pressure, and which enables us to obtain the well-posedness of the transonic shock problem in this case. For the class of nozzles treated in this paper without additional slowly-varying assumption, it seems to be difficult to adapt the previous ideas and analysis in [3]-[4], [9], [15]-[16] directly. Thus we will look for a new approach to determine the position of the transonic shock together with the downstream subsonic flow simultaneously so that the transonic shock problem described by Courant-Friedrichs is well-posed. The main ideas are as follows. First, the steady Euler system in the subsonic domain with a free boundary (the shock curve) can be reformulated as a system consisting of an ordinary differential equation for the shock with a free initial position, a first order nonlinear elliptic system for the pressure and angular velocity, and two transport equations for the specific entropy and Bernoulli's function respectively. We solve such a system through an elaborate iteration scheme by linearizing the nonlinear equations and related nonlinear boundary conditions to obtain several linear systems with some suitable boundary conditions. The key problem in this iteration scheme is to solve a boundary value problem for a first order $2 \times 2$ elliptic system with non-local terms (due to the common actions of the supersonic incoming flow and subsonic flow through R-H conditions) and an unknown parameter (the free position of the shock curve on the wall of the nozzle), see (3.8) in §3. From this, the contractible mappings on the downstream subsonic solutions and the shock curve can be constructed and established. Thus, the proof on Theorem 1.1 can be completed. It should be mentioned that our approach is influenced by the work in [10] and [12], yet the analysis there cannot be applied here.

In what follows, we will use the following convention:

$O(\kappa)$ means that there exists a generic constant $C>0$ independent of $\varepsilon$ and $\kappa$ such that the function $O(\kappa)$ satisfies $\|O(\kappa)\|_{C^{1, \alpha}} \leq C \kappa$. 


\section{The reformulation on problem (1.1) with (1.2)-(1.5).}

In this section, we reformulate the nonlinear problem (1.1) with (1.2)-(1.5) so that we can obtain an algebraic equation for the radial velocity $U_{1}^{+}(x)$, a first order elliptic system for the pressure $P^{+}(x)$ and the angular velocity $U_{2}^{+}(x)$, a first order partial differential equation for the special entropy $S^{+}(x)$. To this end, it is more convenient to use the polar coordinates, $\left(x_{1}, x_{2}\right)=(r \cos \theta, \sin \theta)$, and decompose the velocity $\left(u_{1}^{+}, u_{2}^{+}\right)$into the radial speed $U_{1}^{+}$and angular speed $U_{2}^{+}$as follows

$$
u_{1}^{+}=U_{1}^{+} \cos \theta-U_{2}^{+} \sin \theta, \quad u_{2}^{+}=U_{1}^{+} \sin \theta+U_{2}^{+} \cos \theta .
$$

In the polar coordinates, the domains $\Omega=\left\{\left(x_{1}, x_{2}\right): X_{0}<\sqrt{x_{1}^{2}+x_{2}^{2}}<X_{0}+\right.$ $\left.1,\left|x_{2}\right|<x_{1} \tan \theta_{0}\right\}$ and $\Omega_{+}=\left\{\left(x_{1}, x_{2}\right): \eta\left(x_{2}\right)<x_{1}<\sqrt{\left(X_{0}+1\right)^{2}-x_{2}^{2}},\left|x_{2}\right|<\right.$ $\left.x_{1} \tan \theta_{0}\right\}$ are changed into

$$
R=\left\{(r, \theta): X_{0}<r<X_{0}+1,-\theta_{0}<\theta<\theta_{0}\right\}
$$

and

$$
R_{+}=\left\{(r, \theta): \xi(\theta)<r<X_{0}+1,-\theta_{0}<\theta<\theta_{0}\right\}
$$

respectively, where $r=\xi(\theta)$ stands for the equation of shock curve $\Sigma$ in the polar coordinate $(r, \theta)$.

It follows a direct computation that (1.1) and (1.2) become respectively

$$
\left\{\begin{array}{l}
\partial_{r}\left(\rho U_{1}\right)+\frac{1}{r} \partial_{\theta}\left(\rho U_{2}\right)+\frac{\rho U_{1}}{r}=0 \\
U_{1} \partial_{r} U_{1}+\frac{U_{2}}{r} \partial_{\theta} U_{1}+\frac{\partial_{r} P}{\rho}-\frac{U_{2}^{2}}{r}=0 \\
U_{1} \partial_{r} U_{2}+\frac{U_{2}}{r} \partial_{\theta} U_{2}+\frac{1}{r} \frac{\partial_{\theta} P}{\rho}+\frac{U_{1} U_{2}}{r}=0 \\
U_{1} \partial_{r} S+\frac{U_{2}}{r} \partial_{\theta} S=0
\end{array}\right.
$$

and

$$
\left\{\begin{array}{l}
{\left[\rho U_{1}\right]-\frac{\xi^{\prime}(\theta)}{\xi(\theta)}\left[\rho U_{2}\right]=0,} \\
{[\rho U \otimes U+P I] \cdot\left(1,-\frac{\xi^{\prime}(\theta)}{\xi(\theta)}\right)=0,} \\
{\left[\left(\rho e+\frac{1}{2} \rho|U|^{2}+P\right) U_{1}\right]-\frac{\xi^{\prime}(\theta)}{\xi(\theta)}\left[\left(\rho e+\frac{1}{2} \rho|U|^{2}+P\right) U_{2}\right]=0,}
\end{array}\right.
$$

where $U=\left(U_{1}, U_{2}\right)$.

Meanwhile, (1.4) and (1.5) are converted into

$$
U_{2}^{+}\left(r, \pm \theta_{0}\right)=0
$$

and

$$
P^{+}\left(X_{0}+1, \theta\right)=P_{e}+\varepsilon P_{0}(\theta)
$$

with the compatible condition

$$
P_{0}^{\prime}\left( \pm \theta_{0}\right)=0 .
$$

In addition, it follows from (2.5) and a direct computation that on $r=\xi(\theta)$

$$
\left(P-P_{0}^{+}\left(r_{0}\right), S-S_{0}^{+}\right)=\left(\tilde{g}_{1}, \tilde{g}_{2}\right)\left(\omega^{2}, P_{0}^{-}-P_{0}^{-}\left(r_{0}\right), U_{0}^{-}-U_{0}^{-}\left(r_{0}\right)\right)
$$

here $\tilde{g}_{i}(0,0,0)=0$ for $i=1,2$ and $\omega=\frac{U_{2}}{U_{1}}$. 
Furthermore, it follows from the third equation in (2.5) that $r=\xi(\theta)$ satisfies

$$
\xi^{\prime}(\theta)=\xi(\theta)\left(\frac{\rho U_{1}^{2} \omega}{[P]+\rho U_{1}^{2} \omega^{2}}\right)(\xi(\theta), \theta)
$$

We now decompose the elliptic-hyperbolic system (2.4) by its elliptic and hyperbolic modes.

It can be checked [9] that $\omega=\frac{U_{2}}{U_{1}}$ and $P$ satisfy:

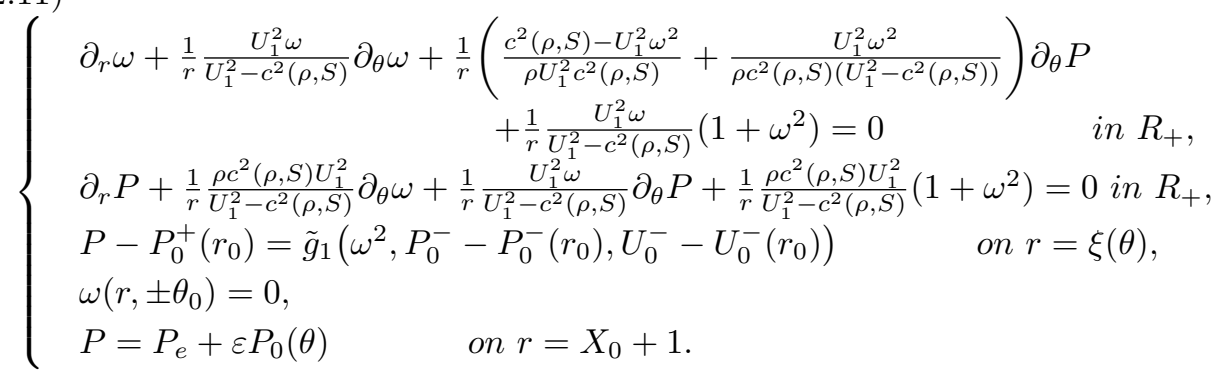

In addition, it follows from the fourth equation in (2.4), (2.9) and the Bernoulli's law that

$$
\left\{\begin{array}{l}
U_{1} \partial_{r} S+\frac{U_{2}}{r} \partial_{\theta} S=0 \quad \text { in } \quad R_{+} \\
S-S_{0}^{+}=\tilde{g}_{2}\left(\omega^{2}, P_{0}^{-}-P_{0}^{-}\left(r_{0}\right), U_{0}^{-}-U_{0}^{-}\left(r_{0}\right)\right)
\end{array} \quad \text { on } \quad r=\xi(\theta)\right.
$$

and

$$
U_{1}^{2}\left(1+\omega^{2}\right)+\frac{2 \gamma P}{(\gamma-1) \rho}=C_{0} \equiv\left(U_{0}^{-}\left(X_{0}\right)\right)^{2}+\frac{2 \gamma P_{0}^{-}\left(X_{0}\right)}{(\gamma-1) \rho_{0}^{-}\left(X_{0}\right)}
$$

Thus, to prove Theorem 1.1, it suffices to solve the problems (2.10)-(2.13). To this end, it is convenient to reduce the free boundary problem (2.10)-(2.13) into a fixed boundary value problem by setting

$$
z_{1}=\frac{r-\xi(\theta)}{X_{0}+1-\xi(\theta)}\left(X_{0}+1-r_{0}\right), \quad z_{2}=\theta
$$

Then the domain $R_{+}$defined in (2.3) is transformed into

$$
E_{+}=\left\{\left(z_{1}, z_{2}\right): 0<z_{1}<X_{0}+1-r_{0},-\theta_{0}<z_{2}<\theta_{0}\right\}
$$

Thus, the problems (2.10)-(2.12) can be rewritten respectively as

$$
\xi^{\prime}\left(z_{2}\right)=\frac{\xi\left(z_{2}\right)\left(\rho U_{1}^{2} \omega\right)\left(\xi\left(z_{2}\right), z_{2}\right)}{P\left(\xi\left(z_{2}\right), z_{2}\right)-P_{0}^{-}\left(\xi\left(z_{2}\right)\right)+\left(\rho U_{1}^{2} \omega^{2}\right)\left(\xi\left(z_{2}\right), z_{2}\right)} \quad \text { in } \quad\left[-\theta_{0}, \theta_{0}\right]
$$


and

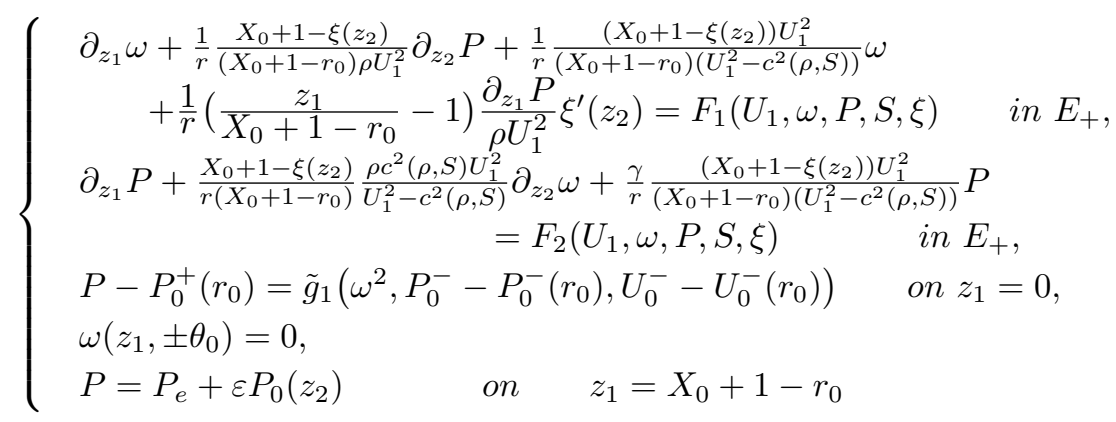

and

(2.18)

$$
\left\{\begin{array}{l}
\left(\left(X_{0}+1-r_{0}\right) \xi\left(z_{2}\right)+\left(X_{0}+1-\xi\left(z_{2}\right)\right) z_{1}+\left(z_{1}-\left(X_{0}+1-r_{0}\right)\right) \xi^{\prime}\left(z_{2}\right) \omega\right) \partial_{z_{1}} S \\
\quad+\left(X_{0}+1-\xi\left(z_{2}\right)\right) \omega \partial_{z_{2}} S=0 \quad \text { in } \quad E_{+}, \\
S-S_{0}^{+}=\tilde{g}_{2}\left(\omega^{2}, P_{0}^{-}-P_{0}^{-}\left(r_{0}\right), U_{0}^{-}-U_{0}^{-}\left(r_{0}\right)\right),
\end{array}\right.
$$

where $r=\xi\left(z_{2}\right)+\frac{X_{0}+1-\xi\left(z_{2}\right)}{X_{0}+1-r_{0}} z_{1}, F_{1}$ and $F_{2}$ are error terms of second order.

We now set for $z \in E_{+}$

$$
\left\{\begin{array}{l}
\left(U_{1}, \omega, P, S\right)(z)=\left(U_{1}, \omega, P, S\right)\left(\xi\left(z_{2}\right)+\frac{X_{0}+1-\xi\left(z_{2}\right)}{X_{0}+1-r_{0}} z_{1}, z_{2}\right) \\
\tilde{U}_{0}^{+}\left(z_{1}\right)=U_{0}^{+}\left(r_{0}+z_{1}\right), \quad \tilde{P}_{0}^{+}\left(z_{1}\right)=P_{0}^{+}\left(r_{0}+z_{1}\right)
\end{array}\right.
$$

and

(2.19) $W(z)=\left(W_{1}, W_{2}, W_{3}, W_{4}, W_{5}\right)(z)=\left(U_{1}-\tilde{U}_{0}^{+}, \omega, P-\tilde{P}_{0}^{+}, S-S_{0}^{+}, \xi-r_{0}\right)(z)$.

Then through a direct but tedious computation, one can derive from (2.16)-(2.17) that

$$
W_{5}^{\prime}\left(z_{2}\right)=\frac{\xi\left(z_{2}\right) \rho\left(0, z_{2}\right)\left(U_{1}\left(0, z_{2}\right)\right)^{2} W_{2}\left(0, z_{2}\right)}{P\left(0, z_{2}\right)-P_{0}^{-}\left(\xi\left(z_{2}\right)\right)+\rho\left(0, z_{2}\right) U_{1}^{2}\left(0, z_{2}\right) \omega^{2}\left(0, z_{2}\right)}
$$

and

$$
\left\{\begin{array}{c}
\partial_{z_{1}} W_{2}+\frac{1}{\left(r_{0}+z_{1}\right) \tilde{\rho}_{0}^{+}\left(\tilde{U}_{0}^{+}\right)^{2}} \partial_{z_{2}} W_{3}+\frac{1}{r_{0}+z_{1}} \frac{\left(\tilde{U}_{0}^{+}\right)^{2}}{\left(\tilde{U}_{0}^{+}-c^{2}\left(\tilde{\rho}_{0}^{+}, S_{0}^{+}\right)\right.} W_{2} \\
\quad-\frac{1}{r_{0}+z_{1}}\left(1-\frac{z_{1}}{X_{0}+1-r_{0}}\right) \frac{\partial_{z_{1}} \tilde{P}_{0}^{+}}{\tilde{\rho}_{0}^{+}\left(\tilde{U}_{0}^{+}\right)^{2}} W_{5}^{\prime}\left(z_{2}\right)=F_{3}\left(z_{1}, W, \nabla W\right) \quad \text { in } E_{+}, \\
\partial_{z_{1}} W_{3}+\frac{1}{r_{0}+z_{1}} \frac{\tilde{\rho}_{0}^{+} c^{2}\left(\tilde{\rho}_{0}^{+}, \hat{S}_{0}^{+}\right)\left(\tilde{U}_{0}^{+}\right)^{2}}{\left(\tilde{U}_{0}^{+}\right)^{2}-c^{2}\left(\tilde{\rho}_{0}^{+}, S_{0}^{+}\right)} \partial_{z_{2}} W_{2}+\frac{\gamma}{r_{0}+z_{1}} \frac{\left(\tilde{U}_{0}^{+}\right)^{2}}{\left(\tilde{U}_{0}^{+}\right)^{2}-c^{2}\left(\tilde{\rho}_{0}^{+}, S_{0}^{+}\right)} W_{3} \\
\quad+B_{1}\left(z_{1}\right) W_{3}+B_{2}\left(z_{1}\right) W_{4}+B_{3}\left(z_{1}\right) W_{5}=F_{4}\left(z_{1}, W, \nabla W\right)+R_{1}(W) \text { in } E_{+}, \\
W_{3}\left(0, z_{2}\right)=B_{4} W_{5}\left(z_{2}\right)+R_{2}\left(z_{2}\right), \\
W_{3}\left(X_{0}+1-r_{0}, z_{2}\right)=\varepsilon P_{0}\left(z_{2}\right), \quad W_{2}\left(z_{1}, \pm \theta_{0}\right)=0
\end{array}\right.
$$


here $F_{3}, F_{4}$ and $R_{1}, R_{2}$ are error terms of second order with respect to $W$ and/or $\nabla W$, and

$$
\begin{aligned}
& B_{1}\left(z_{1}\right)=\frac{\gamma \tilde{P}_{0}^{+}\left(z_{1}\right)\left(\left(\tilde{U}_{0}^{+}\left(z_{1}\right)\right)^{2}+\frac{2}{\gamma-1} c^{2}\left(\tilde{\rho}_{0}^{+}\left(z_{1}\right), S_{0}^{+}\right)\right)}{\left(r_{0}+z_{1}\right)\left(c^{2}\left(\tilde{\rho}_{0}^{+}\left(z_{1}\right), S_{0}^{+}\right)-\left(\tilde{U}_{0}^{+}\left(z_{1}\right)\right)^{2}\right)^{2}} \frac{\gamma-1}{\tilde{\rho}_{0}^{+}\left(z_{1}\right)}>0, \\
& B_{2}\left(z_{1}\right)=\frac{\gamma \tilde{P}_{0}^{+}\left(z_{1}\right)\left(\left(\tilde{U}_{0}^{+}\left(z_{1}\right)\right)^{2}+\frac{2}{\gamma-1} c^{2}\left(\tilde{\rho}_{0}^{+}\left(z_{1}\right), S_{0}^{+}\right)\right)}{\left(r_{0}+z_{1}\right)\left(c^{2}\left(\tilde{\rho}_{0}^{+}\left(z_{1}\right), S_{0}^{+}\right)-\left(\tilde{U}_{0}^{+}\left(z_{1}\right)\right)^{2}\right)^{2}} \frac{\tilde{P}_{0}^{+}\left(z_{1}\right)}{c_{v} \tilde{\rho}_{0}^{+}\left(z_{1}\right)}>0, \\
& B_{3}\left(z_{1}\right)=\frac{\gamma\left(X_{0}+1\right)\left(\tilde{U}_{0}^{+}\left(z_{1}\right)\right)^{2} \tilde{P}_{0}^{+}\left(z_{1}\right)}{\left(X_{0}+1-r_{0}\right)\left(r_{0}+z_{1}\right)^{2}\left(c^{2}\left(\tilde{\rho}_{0}^{+}\left(z_{1}\right), S_{0}^{+}\right)-\left(\tilde{U}_{0}^{+}\left(z_{1}\right)\right)^{2}\right)}>0, \\
& B_{4}=\frac{(\gamma-1)\left(\rho_{0}^{-} U_{0}^{-}\right)\left(r_{0}\right)}{r_{0}\left(c^{2}\left(\rho_{0}^{+}, S_{0}^{+}\right)-\left(U_{0}^{+}\right)^{2}\right)\left(r_{0}\right)}\left(U_{0}^{+}\left(\left(U_{0}^{+}\right)^{2}+\frac{2}{\gamma-1} c^{2}\left(\rho_{0}^{+}, S_{0}^{+}\right)\right)\right. \\
& \left.R_{1}(W(z))=O\left(|W(z)|^{2}\right), \quad U_{0}^{-}\left(\left(U_{0}^{+}\right)^{2}+\frac{1}{\gamma-1} c^{2}\left(\rho_{0}^{+}, S_{0}^{+}\right)\right)\right)\left(r_{0}\right), \\
& R_{2}\left(z_{2}\right) \equiv R_{2}\left(W\left(0, z_{2}\right)\right)=O\left(\left|W\left(0, z_{2}\right)\right|^{2}\right) .
\end{aligned}
$$

Meanwhile, it follows from (2.18) and (2.13) that

$$
\left\{\begin{array}{l}
\left(\left(X_{0}+1-r_{0}\right) \xi\left(z_{2}\right)+\left(z_{1}-\left(X_{0}+1-r_{0}\right)\right) \xi^{\prime}\left(z_{2}\right)\right. \\
\left.\quad+\left(X_{0}+1-\xi\left(z_{2}\right)\right) z_{1} W_{2}\right) \partial_{z_{1}} W_{4}+\left(X_{0}+1-\xi\left(z_{2}\right)\right) W_{2} \partial_{z_{2}} W_{4}=0 \text { in } E_{+}, \\
W_{4}\left(0, z_{2}\right)=B_{5} W_{5}\left(z_{2}\right)+R_{3}\left(z_{2}\right)
\end{array}\right.
$$

and

$$
W_{1}+\frac{\gamma}{\gamma-1} \frac{1}{\tilde{U}_{0}^{+} \tilde{\rho}_{0}^{+}} W_{3}-\frac{\gamma}{\gamma-1} \frac{\tilde{P}_{0}^{+}}{\tilde{U}_{0}^{+}\left(\tilde{\rho}_{0}^{+}\right)^{2}}\left(\rho-\tilde{\rho}_{0}^{+}\right)=F_{5}(W)
$$

where $B_{5}=\frac{(\gamma-1) c_{v}}{r_{0}} \frac{\left(\rho_{0}^{-} U_{0}^{-}\right)\left(r_{0}\right)}{P_{0}^{+}\left(r_{0}\right)}\left(U_{0}^{-}\left(r_{0}\right)-U_{0}^{+}\left(r_{0}\right)\right)>0, R_{3}\left(z_{2}\right)=R_{3}\left(W\left(0, z_{2}\right)\right)$ and $F_{5}(W)$ in $(2.22)-(2.23)$ are the error terms of second order.

With these, Theorem 1.1 follows from the following conclusion:

Theorem 2.1. Under the assumptions in Theorem 1.1, there exists a positive constant $C$ depending only on $\alpha$ and the supersonic incoming flow such that the system (2.20)-(2.23) has a unique solution $W$ with the following estimates

$$
\sum_{i=1}^{4}\left\|W_{i}\right\|_{C^{2, \alpha}\left(\bar{E}_{+}\right)}+\left\|W_{5}\right\|_{C^{3, \alpha}\left[-\theta_{0}, \theta_{0}\right]} \leq C \varepsilon .
$$

\section{An Iteration Scheme.}

In this section, we construct an iteration scheme to solve the nonlinear problem (2.20)-(2.23). 
To this end, we introduce an iteration space as follows

$$
\begin{aligned}
& \Xi_{\delta}=\left\{W: \sum_{i=1}^{4}\left\|W_{i}\right\|_{C^{2, \alpha}\left(\bar{E}_{+}\right)}+\left\|W_{5}\right\|_{C^{3, \alpha}\left[-\theta_{0}, \theta_{0}\right]} \leq \delta ; \partial_{z_{2}} W_{j}\left(z_{1}, \pm \theta_{0}\right)=0,\right. \\
& \left.j=1,3,4 ; W_{2}\left(z_{1}, \pm \theta_{0}\right)=\partial_{z_{2}}^{2} W_{2}\left(z_{1}, \pm \theta_{0}\right)=0 ; W_{5}^{\prime}\left( \pm \theta_{0}\right)=W_{5}^{(3)}\left( \pm \theta_{0}\right)=0\right\},
\end{aligned}
$$

where the constant $\delta>0$ will be determined later on.

In terms of the notations in (2.19), each $\hat{W} \in \Xi_{\delta}$ has the following expression

$$
\left(\hat{U}_{1}(z), \hat{\omega}(z), \hat{P}(z), \hat{S}(z), \hat{\rho}(z) ; \hat{\xi}\left(z_{2}\right)\right) .
$$

We now define the linearized scheme to the problem (2.20)-(2.23) and determine its corresponding solution $\bar{W}(z)=\left(\bar{W}_{1}(z), \bar{W}_{2}(z), \bar{W}_{3}(z), \bar{W}_{4}(z), \bar{W}_{5}\left(z_{2}\right)\right)$ as follows.

1. The determination of $\bar{W}_{5}$.

Due to $(2.20), \bar{W}_{5}$ is defined as

$$
\bar{W}_{5}^{\prime}\left(z_{2}\right)=\frac{r_{0} \tilde{\rho}_{0}^{+}(0)\left(\tilde{U}_{0}^{+}(0)\right)^{2}}{\tilde{P}_{0}^{+}(0)-P_{0}^{-}\left(r_{0}\right)} \bar{W}_{2}\left(0, z_{2}\right)+F_{6}\left(z_{2}\right)
$$

where

$$
F_{6}\left(z_{2}\right) \equiv\left(\frac{\hat{\xi}\left(z_{2}\right) \hat{\rho}\left(0, z_{2}\right)\left(\hat{U}_{1}\left(0, z_{2}\right)\right)^{2}}{\hat{P}\left(0, z_{2}\right)-P_{0}^{-}(\hat{\xi})+\left(\hat{\rho}\left(\hat{U}_{1}\right)^{2}(\hat{\omega})^{2}\right)\left(0, z_{2}\right)}-\frac{r_{0} \tilde{\rho}_{0}^{+}(0)\left(\tilde{U}_{0}^{+}(0)\right)^{2}}{\tilde{P}_{0}^{+}(0)-P_{0}^{-}\left(r_{0}\right)}\right) \hat{W}_{2}\left(0, z_{2}\right) .
$$

Since $\hat{W} \in \Xi_{\delta}$, one checks easily that

$$
F_{6}\left( \pm \theta_{0}\right)=F_{6}^{\prime \prime}\left( \pm \theta_{0}\right)=0,\left\|F_{6}\right\|_{C^{k, \alpha}\left[-\theta_{0}, \theta_{0}\right]} \leq C \delta\left\|\hat{W}_{2}\right\|_{C^{k, \alpha}\left(\bar{E}_{+}\right)} \text {for } k=0,1,2
$$

here and below the positive generic constant $C$ is independent of $\delta$.

\section{The determination of $\bar{W}_{4}$.}

From (2.22), $\bar{W}_{4}$ is required to satisfy

$$
\begin{gathered}
\left(\left(X_{0}+1-r_{0}\right) \hat{\xi}\left(z_{2}\right)+\left(z_{1}-\left(X_{0}+1-r_{0}\right)\right.\right. \\
\left.\left.+\left(X_{0}+1-\hat{\xi}\left(z_{2}\right)\right) z_{1}\right) \hat{\xi}^{\prime}\left(z_{2}\right) \hat{W}_{2}\right) \partial_{z_{1}} \bar{W}_{4}+\left(X_{0}+1-\hat{\xi}\left(z_{2}\right)\right) \hat{W}_{2} \partial_{z_{2}} \bar{W}_{4}=0 \text { in } E_{+}
\end{gathered}
$$

with the initial data $\bar{W}_{4}\left(0, z_{2}\right)$ being chosen in terms of the expression of $W_{4}\left(0, z_{2}\right)$ in (2.22) (one can see the details in (3.6) below).

Let $z_{2}(s ; \beta)$ be the characteristics going through $z=\left(z_{1}, z_{2}\right)$ with $z_{2}(0 ; \beta)=\beta$ for the first order differential equation (3.5). It is noted that the variable $\beta$ can be also regarded as the function of $z=\left(z_{1}, z_{2}\right)$, which is denoted by $\beta=\beta(z)$. Then it follows from a direct computation that

$$
\bar{W}_{4}(z)=\bar{W}_{4}(0, \beta(z))=B_{5} \bar{W}_{5}\left(z_{2}\right)+F_{7}(z),
$$

where

$$
F_{7}(z) \equiv F_{7}(\hat{W})(z)=B_{5} \int_{z_{2}}^{\beta(z)} \hat{W}_{5}^{\prime}(s) d s+R_{3}(\hat{W}(0, \beta(z)))
$$


Moreover, we have

$$
\begin{aligned}
& \partial_{z_{2}} F_{7}\left(z_{1}, \pm \theta_{0}\right)=0 \\
& \left\|F_{7}\right\|_{C^{k, \alpha}\left(\bar{E}_{+}\right)} \leq C \delta\left(\sum_{l=1}^{4}\left\|\hat{W}_{l}\right\|_{C^{k, \alpha}\left(\bar{E}_{+}\right)}+\left\|\hat{W}_{5}\right\|_{C^{k+1, \alpha}\left[-\theta_{0}, \theta_{0}\right]}\right) \text { for } k=0,1,2 .
\end{aligned}
$$

3. The determinations of $\bar{W}_{2}, \bar{W}_{3}$ and $\bar{W}_{5}\left(-\theta_{0}\right)$.

By (2.21), (3.3), (3.6) and (2.9), in terms of the unknown shock position $\bar{W}_{5}\left(-\theta_{0}\right)$ at the nozzle wall $\theta=-\theta_{0}$ (it should be noted that $\bar{W}_{5}\left(-\theta_{0}\right)$ will be determined together with the solution $\bar{W}_{2}$ and $\bar{W}_{3}$ of the linearized equations), we define $\bar{W}_{2}$ and $\bar{W}_{3}$ by solving the following problem

$$
\left\{\begin{array}{c}
\partial_{z_{1}} \bar{W}_{2}+\frac{1}{\left(r_{0}+z_{1}\right) \tilde{\rho}_{0}^{+}\left(\tilde{U}_{0}^{+}\right)^{2}} \partial_{z_{2}} \bar{W}_{3}+\frac{1}{r_{0}+z_{1}} \frac{\left(\tilde{U}_{0}^{+}\right)^{2}}{\left(\tilde{U}_{0}^{+}\right)^{2}-c^{2}\left(\tilde{\rho}_{0}^{+}, S_{0}^{+}\right)} \bar{W}_{2} \\
-\frac{1}{r_{0}+z_{1}}\left(1-\frac{z_{1}}{X_{0}+1-r_{0}}\right) \frac{\partial_{z_{1}} \tilde{P}_{0}^{+}}{\tilde{\rho}_{0}^{+}\left(\tilde{U}_{0}^{+}\right)^{2}} \frac{r_{0} \tilde{\rho}_{0}^{+}(0)\left(\tilde{U}_{0}^{+}(0)\right)^{2}}{\tilde{P}_{0}^{+}(0)-P^{-}\left(r_{0}\right)} \bar{W}_{2}\left(0, z_{2}\right) \\
=F_{3}\left(z_{1}, \hat{W}, \nabla W\right)+\frac{1}{r_{0}+z_{1}}\left(1-\frac{z_{1}}{X_{0}+1-r_{0}}\right) \frac{\partial_{z_{1}} \tilde{P}_{0}^{+}}{\tilde{\rho}_{0}^{+}\left(\tilde{U}_{0}^{+}\right)^{2}} F_{6}\left(z_{2}\right) \quad \text { in } \quad E_{+}, \\
\partial_{z_{1}} \bar{W}_{3}+\frac{1}{r_{0}+z_{1}} \frac{\tilde{\rho}_{0}^{+} c^{2}\left(\tilde{\rho}_{0}^{+}, S_{0}^{+}\right)\left(\tilde{U}_{0}^{+}\right)^{2}}{\left(\tilde{U}_{0}^{+}\right)^{2}-c^{2}\left(\tilde{\rho}_{0}^{+}, S_{0}^{+}\right)} \partial_{z_{2}} \bar{W}_{2}+\left(\frac{\gamma}{r_{0}+z_{1}} \frac{\left(\tilde{U}_{0}^{+}\right)^{2}}{\left(\tilde{U}_{0}^{+}\right)^{2}-c^{2}\left(\tilde{\rho}_{0}^{+}, S_{0}^{+}\right)}+B_{1}\left(z_{1}\right)\right) \bar{W}_{3} \\
+\left(B_{5} B_{2}\left(z_{1}\right)+B_{3}\left(z_{1}\right)\right)\left(\bar{W}_{5}\left(-\theta_{0}\right)+\frac{r_{0} \tilde{\rho}_{0}^{+}(0)\left(\tilde{U}_{0}^{+}(0)\right)^{2}}{\tilde{P}_{0}^{+}(0)-P_{0}^{-}\left(r_{0}\right)} \int_{-\theta_{0}}^{z_{2}} \bar{W}_{2}(0, s) d s\right) \\
=F_{4}\left(z_{1}, \hat{W}, \nabla \hat{W}\right)+R_{1}(\hat{W})-\left(B_{5} B_{2}\left(z_{1}\right)+B_{3}\left(z_{1}\right)\right) \int_{-\theta_{0}}^{z_{2}} F_{6}(s) d s \\
-B_{2}\left(z_{1}\right) F_{7}(z) \quad \operatorname{in} E_{+}, \\
\bar{W}_{3}\left(0, z_{2}\right)=B_{4}\left(\bar{W}_{5}\left(-\theta_{0}\right)+\frac{r_{0} \tilde{o}_{0}^{+}(0)\left(\tilde{U}_{0}^{+}(0)\right)^{2}}{\tilde{P}_{0}^{+}(0)-P_{0}^{-}\left(r_{0}\right)} \int_{-\theta_{0}}^{z_{2}} \bar{W}_{2}(0, s) d s\right) \\
\quad+B_{4} \int_{-\theta_{0}}^{z_{2}} F_{6}(s) d s+R_{2}\left(\hat{W}\left(0, z_{2}\right)\right) \\
\bar{W}_{3}\left(X_{0}+1-r_{0}, z_{2}\right)=\varepsilon P_{0}\left(z_{2}\right), \bar{W}_{2}\left(z_{1}, \pm \theta_{0}\right)=0 .
\end{array}\right.
$$

To write the first and second equations of (3.8) in divergence forms and for notational conveniences, we define

$$
\begin{aligned}
\lambda_{1}\left(z_{1}\right) & =\exp \left(\int_{0}^{z_{1}} \frac{1}{r_{0}+s} \frac{\left(\tilde{U}_{0}^{+}\right)^{2}(s)}{\left(\tilde{U}_{0}^{+}\right)^{2}(s)-c^{2}\left(\tilde{\rho}_{0}^{+}, S_{0}^{+}\right)(s)} d s\right)>0, \\
\lambda_{2}\left(z_{1}\right) & =\frac{1}{\left(r_{0}+z_{1}\right) \tilde{\rho}_{0}^{+}\left(\tilde{U}_{0}^{+}\right)^{2}} \lambda_{1}\left(z_{1}\right)>0, \\
\lambda_{3}\left(z_{1}\right) & =\frac{1}{r_{0}+z_{1}}\left(1-\frac{z_{1}}{X_{0}+1-r_{0}}\right) \frac{\partial_{z_{1}} \tilde{P}_{0}^{+}}{\tilde{\rho}_{0}^{+}\left(\tilde{U}_{0}^{+}\right)^{2}} \frac{r_{0} \tilde{\rho}_{0}^{+}(0)\left(\tilde{U}_{0}^{+}(0)\right)^{2}}{\tilde{P}_{0}^{+}(0)-P_{0}^{-}\left(r_{0}\right)} \lambda_{1}\left(z_{1}\right) \geq 0 \\
\text { with } \lambda_{3}\left(X_{0}+1-r_{0}\right)=0, \quad\left(\tilde{U}_{0}^{+}\right)^{2}(s) & \\
\lambda_{4}\left(z_{1}\right) & =\exp \left(\int_{0}^{z_{1}}\left(\frac{\gamma}{r_{0}+s} \frac{\left.\left.\tilde{U}_{0}(s)\right) d s\right)>0,}{\left(\tilde{U}_{0}^{+}\right)^{2}(s)-c^{2}\left(\tilde{\rho}_{0}^{+}, S_{0}^{+}\right)(s)}+B_{1}\right)\right. \\
\lambda_{5}\left(z_{1}\right) & =\frac{1}{r_{0}+z_{1}} \frac{\tilde{\rho}_{0}^{+} c^{2}\left(\tilde{\rho}_{0}^{+}, S_{0}^{+}\right)\left(\tilde{U}_{0}^{+}\right)^{2}}{c^{2}\left(\tilde{\rho}_{0}^{+}, S_{0}^{+}\right)-\left(\tilde{U}_{0}^{+}\right)^{2}} \lambda_{4}\left(z_{1}\right)>0, \\
\lambda_{6}\left(z_{1}\right) & =\left(B_{5} B_{2}\left(z_{1}\right)+B_{3}\left(z_{1}\right)\right) \lambda_{4}\left(z_{1}\right)>0, \quad \lambda_{7}=\frac{r_{0} \tilde{\rho}_{0}^{+}(0)\left(\tilde{U}_{0}^{+}(0)\right)^{2}}{\tilde{P}_{0}^{+}(0)-P_{0}^{-}\left(r_{0}\right)}>0
\end{aligned}
$$


and

$$
\left\{\begin{array}{c}
G_{1}(z)=\lambda_{1}\left(z_{1}\right)\left(F_{3}\left(z_{1}, \hat{W}, \nabla \hat{W}\right)+\frac{X_{0}+1-r_{0}-z_{1}}{\left(r_{0}+z_{1}\right)\left(X_{0}+1-r_{0}\right)} \frac{\partial_{z_{1}} \tilde{P}_{0}^{+}}{\tilde{\rho}_{0}^{+}\left(\tilde{U}_{0}^{+}\right)^{2}} F_{6}\left(z_{2}\right)\right), \\
G_{2}(z)=\lambda_{4}\left(z_{1}\right)\left(F_{4}\left(z_{1}, \hat{W}, \nabla \hat{W}\right)+R_{1}(\hat{W})\right. \\
\left.\quad-\left(B_{5} B_{2}\left(z_{1}\right)+B_{3}\left(z_{1}\right)\right) \int_{-\theta_{0}}^{z_{2}} F_{6}(s) d s-B_{4}\left(z_{1}\right) F_{7}(z)\right), \\
G_{3}\left(z_{2}\right)=B_{4} \int_{-\theta_{0}}^{z_{2}} F_{6}(s) d s+R_{2}\left(\hat{W}\left(0, z_{2}\right)\right) .
\end{array}\right.
$$

It follows from (3.4), (3.7) and a direct computation that

$$
\left\{\begin{array}{l}
G_{1}\left(z_{1}, \pm \theta_{0}\right)=0, \quad \partial_{z_{2}} G_{2}\left(z_{1}, \pm \theta_{0}\right)=0, \\
\left\|\left(G_{1}, G_{2}\right)\right\|_{C^{k-1, \alpha}\left(\bar{E}_{+}\right)} \leq C \delta\|\hat{W}\|_{C^{k, \alpha}\left(\bar{E}_{+}\right)}, \quad k=1,2
\end{array}\right.
$$

and

$$
\partial_{z_{2}} G_{3}\left( \pm \theta_{0}\right)=0, \quad\left\|G_{3}\right\|_{C^{k, \alpha}\left[-\theta_{0}, \theta_{0}\right]} \leq C \delta\|\hat{W}\|_{C^{k, \alpha}\left(\bar{E}_{+}\right)} \quad \text { for } k=0,1,2 .
$$

Then, direct computations show (3.8) can be easily rewritten as

$$
\left\{\begin{array}{c}
\partial_{z_{1}}\left(\lambda_{1}\left(z_{1}\right) \bar{W}_{2}\right)+\partial_{z_{2}}\left(\lambda_{2}\left(z_{1}\right) \bar{W}_{3}-\lambda_{3}\left(z_{1}\right)\left(\frac{\bar{W}_{5}\left(-\theta_{0}\right)}{\lambda_{7}}\right.\right. \\
\left.\left.\quad+\int_{-\theta_{0}}^{z_{2}} \bar{W}_{2}(0, s) d s\right)-\int_{-\theta_{0}}^{z_{2}} G_{1}\left(z_{1}, s\right) d s\right)=0 \\
\partial_{z_{1}}\left(\lambda_{4}\left(z_{1}\right) \bar{W}_{3}\right)-\partial_{z_{2}}\left(\lambda_{5}\left(z_{1}\right) \bar{W}_{2}\right) \\
+\lambda_{6}\left(z_{1}\right)\left(\bar{W}_{5}\left(-\theta_{0}\right)+\lambda_{7} \int_{-\theta_{0}}^{z_{2}} \bar{W}_{2}(0, s) d s\right)=G_{2}(z) \\
\bar{W}_{3}\left(0, z_{2}\right)=B_{4}\left(\bar{W}_{5}\left(-\theta_{0}\right)+\lambda_{7} \int_{-\theta_{0}}^{z_{2}} \bar{W}_{2}(0, s) d s\right)+G_{3}\left(z_{2}\right) \\
\bar{W}_{3}\left(X_{0}+1-r_{0}, z_{2}\right)=\varepsilon P_{0}\left(z_{2}\right), \quad \bar{W}_{2}\left(z_{1}, \pm \theta_{0}\right)=0
\end{array}\right.
$$

In order to solve the problem (3.11), our approach is to introduce a potential function $\phi(z)$ such that (3.11) can be reduced into a second order elliptic equation with some suitable boundary conditions.

Indeed, by the first equation in (3.11), one can set

$$
\left\{\begin{array}{l}
\partial_{z_{1}} \phi=\lambda_{2}\left(z_{1}\right) \bar{W}_{3}-\lambda_{3}\left(z_{1}\right)\left(\frac{\bar{W}_{5}\left(-\theta_{0}\right)}{\lambda_{7}}+\int_{-\theta_{0}}^{z_{2}} \bar{W}_{2}(0, s) d s\right)-\int_{-\theta_{0}}^{z_{2}} G_{1}\left(z_{1}, s\right) d s \\
\partial_{z_{2}} \phi=-\lambda_{1}\left(z_{1}\right) \bar{W}_{2}, \quad \phi\left(0,-\theta_{0}\right)=0 .
\end{array}\right.
$$

Since $\lambda_{1}(0)=1$, one has

$$
\left\{\begin{array}{l}
\bar{W}_{2}=-\frac{1}{\lambda_{1}\left(z_{1}\right)} \partial_{z_{2}} \phi \\
\bar{W}_{3}=\frac{\partial_{z_{1}} \phi}{\lambda_{2}\left(z_{1}\right)}-\frac{\lambda_{3}\left(z_{1}\right)}{\lambda_{2}\left(z_{1}\right)}\left(\phi\left(0, z_{2}\right)-\frac{\bar{W}_{5}\left(-\theta_{0}\right)}{\lambda_{7}}\right)+\frac{1}{\lambda_{2}\left(z_{1}\right)} \int_{-\theta_{0}}^{z_{2}} G_{1}\left(z_{1}, s\right) d s .
\end{array}\right.
$$

Substituting (3.13) into the second equation in (3.11) yields

$$
\begin{aligned}
& \partial_{z_{1}}\left(\frac{\lambda_{4}\left(z_{1}\right)}{\lambda_{2}\left(z_{1}\right)} \partial_{z_{1}} \phi\right)-\left(\lambda_{6}\left(z_{1}\right) \lambda_{7}+\frac{d}{d z_{1}}\left(\frac{\lambda_{3}\left(z_{1}\right) \lambda_{4}\left(z_{1}\right)}{\lambda_{2}\left(z_{1}\right)}\right)\right)\left(\phi\left(0, z_{2}\right)-\frac{\bar{W}_{5}\left(-\theta_{0}\right)}{\lambda_{7}}\right) \\
& \quad+\partial_{z_{2}}\left(\frac{\lambda_{5}\left(z_{1}\right)}{\lambda_{1}\left(z_{1}\right)} \partial_{z_{2}} \phi\right)=G_{2}(z)-\partial_{z_{1}}\left(\frac{\lambda_{4}\left(z_{1}\right)}{\lambda_{2}\left(z_{1}\right)} \int_{-\theta_{0}}^{z_{2}} G_{1}\left(z_{1}, s\right) d s\right)
\end{aligned}
$$

Next, we study the boundary conditions for $\phi(z)$. 
It follows from the boundary condition for $\bar{W}_{3}\left(0, z_{2}\right)$ in (3.11) and (3.12) that

$$
\bar{W}_{3}\left(0, z_{2}\right)=-B_{4} \lambda_{7}\left(\phi\left(0, z_{2}\right)-\frac{\bar{W}_{5}\left(-\theta_{0}\right)}{\lambda_{7}}\right)+G_{3}\left(z_{2}\right) .
$$

This, together with (3.13), yields

$$
\begin{aligned}
& \partial_{z_{1}} \phi\left(0, z_{2}\right)+\left(B_{4} \lambda_{7} \lambda_{2}(0)-\lambda_{3}(0)\right)\left(\phi\left(0, z_{2}\right)-\frac{\bar{W}_{5}\left(-\theta_{0}\right)}{\lambda_{7}}\right) \\
= & \lambda_{2}(0) G_{3}\left(z_{2}\right)-\int_{-\theta_{0}}^{z_{2}} G_{1}(0, s) d s .
\end{aligned}
$$

It follows from $\bar{W}_{3}\left(X_{0}+1-r_{0}, z_{2}\right)=\varepsilon P_{0}\left(z_{2}\right),(3.13)$, and $\lambda_{3}\left(X_{0}+1-r_{0}\right)=0$ that

$$
\partial_{z_{1}} \phi=\varepsilon \lambda_{2}\left(X_{0}+1-r_{0}\right) P_{0}\left(z_{2}\right)-\int_{-\theta_{0}}^{z_{2}} G_{1}\left(X_{0}+1-r_{0}, s\right) d s \text { on } z_{1}=X_{0}+1-r_{0} .
$$

In addition, by $\bar{W}_{2}\left(z_{1}, \pm \theta_{0}\right)=0$, one has

$$
\partial_{z_{2}} \phi\left(z_{1}, \pm \theta_{0}\right)=0 \text {. }
$$

Therefore, (3.11) is equivalent to the following problem for a second order non-local elliptic equation for $\phi(z)$ with the unknown constant $\bar{W}_{5}\left(-\theta_{0}\right)$

$$
\left\{\begin{array}{c}
\partial_{z_{1}}\left(\frac{\lambda_{4}\left(z_{1}\right)}{\lambda_{2}\left(z_{1}\right)} \partial_{z_{1}} \phi\right)-\left(\lambda_{6}\left(z_{1}\right) \lambda_{7}+\frac{d}{d z_{1}}\left(\frac{\lambda_{3}\left(z_{1}\right) \lambda_{4}\left(z_{1}\right)}{\lambda_{2}\left(z_{1}\right)}\right)\right)\left(\phi\left(0, z_{2}\right)-\frac{\bar{W}_{5}\left(-\theta_{0}\right)}{\lambda_{7}}\right) \\
+\partial_{z_{2}}\left(\frac{\lambda_{5}\left(z_{1}\right)}{\lambda_{1}\left(z_{1}\right)} \partial_{z_{2}} \phi\right)=G_{2}(z)-\partial_{z_{1}}\left(\frac{\lambda_{4}\left(z_{1}\right)}{\lambda_{2}\left(z_{1}\right)} \int_{-\theta_{0}}^{z_{2}} G_{1}\left(z_{1}, s\right) d s\right) i n E_{+} \\
\partial_{z_{1}} \phi\left(0, z_{2}\right)+\left(B_{4} \lambda_{7} \lambda_{2}(0)-\lambda_{3}(0)\right)\left(\phi\left(0, z_{2}\right)-\frac{\bar{W}_{5}\left(-\theta_{0}\right)}{\lambda_{7}}\right) \\
=\lambda_{2}(0) G_{3}\left(z_{2}\right)-\int_{-\theta_{0}}^{z_{2}} G_{1}(0, s) d s \\
\partial_{z_{1}} \phi=\varepsilon \lambda_{2}\left(X_{0}+1-r_{0}\right) P_{0}\left(z_{2}\right)-\int_{-\theta_{0}}^{z_{2}} G_{1}\left(X_{0}+1-r_{0}, s\right) d s \\
\text { on } z_{1}=X_{0}+1-r_{0}, \\
\partial_{z_{2}} \phi\left(z_{1}, \pm \theta_{0}\right)=0, \quad \phi\left(0,-\theta_{0}\right)=0 .
\end{array}\right.
$$

As will be shown in the next section, (3.18) can be solved under the conditions

$$
\lambda_{6}\left(z_{1}\right) \lambda_{7}+\frac{d}{d z_{1}}\left(\frac{\lambda_{3}\left(z_{1}\right) \lambda_{4}\left(z_{1}\right)}{\lambda_{2}\left(z_{1}\right)}\right)>0 \quad \text { and } \quad B_{4} \lambda_{7} \lambda_{2}(0)-\lambda_{3}(0)<0,
$$

which can be verified by a direct computation in $\S 5$. Details will be given in $\S 4$ and the first part of $\S 5$.

\section{The determination of $\bar{W}_{1}$.}

By (2.23), we set

$$
\bar{W}_{1}=-\frac{\gamma}{(\gamma-1) \tilde{U}_{0}^{+}\left(z_{1}\right) \tilde{\rho}_{0}^{+}\left(z_{1}\right)} \bar{W}_{3}+\frac{\gamma \tilde{P}_{0}^{+}}{(\gamma-1) \tilde{U}_{0}^{+}\left(z_{1}\right)\left(\tilde{\rho}_{0}^{+}\left(z_{1}\right)\right)^{2}}\left(\bar{\rho}-\tilde{\rho}_{0}^{+}\right)+F_{5}(\hat{W}),
$$

where $F_{5}(z)=F_{5}(\hat{W}(z))$ satisfies

$$
\partial_{z_{2}} F_{5}\left(z_{1}, \pm \theta_{0}\right)=0, \quad\left\|F_{5}\right\|_{C^{k, \alpha}\left(\bar{E}_{+}\right)} \leq C \delta\|\hat{W}\|_{C^{k, \alpha}\left(\bar{E}_{+}\right)} \quad \text { for } k=0,1,2
$$

In next section, we focus on solving the first order elliptic system (3.8) which involves the nonlocal term $W_{2}\left(0, z_{2}\right)$ and an unknown constant $W_{5}\left(-\theta_{0}\right)$. 


\section{On a non-local second order elliptic equation with a free parameter.}

In this section, we solve the system (3.8), which is equivalent to (3.11). By the reduction procedure given in $\S 3$, it suffices to solve the boundary value problem (3.18) and derive some desired estimates. To this end, set

$$
E=\left\{\left(z_{1}, z_{2}\right): 0<z_{1}<1,0<z_{2}<1\right\} .
$$

We consider the following boundary value problem for a general second order elliptic equation with a non-local term and an unknown constant

$$
\left\{\begin{array}{l}
\partial_{z_{1}}\left(a_{1}\left(z_{1}\right) \partial_{z_{1}} u\right)+\partial_{z_{2}}\left(a_{2}\left(z_{1}\right) \partial_{z_{2}} u\right)-a_{3}\left(z_{1}\right)\left(u\left(0, z_{2}\right)+\kappa\right)=\partial_{z_{1}} f \text { in } E \\
\partial_{z_{1}} u\left(0, z_{2}\right)-a_{4}\left(u\left(0, z_{2}\right)+\kappa\right)=g_{1}\left(z_{2}\right) \\
\partial_{z_{1}} u\left(1, z_{2}\right)=g_{2}\left(z_{2}\right) \\
\partial_{z_{2}} u\left(z_{1}, 0\right)=\partial_{z_{2}} u\left(z_{1}, 1\right)=u(0,0)=0
\end{array}\right.
$$

where the smooth functions $a_{i}\left(z_{1}\right)(i=1,2,3)$ and the constant $a_{4}$ satisfy $a_{10} \geq a_{j} \geq$ $a_{20}>0(1 \leq j \leq 4)$ for some positive constants $a_{10}$ and $a_{20}$. Here $\kappa$ is a constant to be determined together with the solution $u(z)$ itself.

We first consider the uniqueness of weak solutions to its homogenous problem.

Lemma 4.1. If a pair $(u, \kappa)$ with $u \in H^{1}(E)$ is a weak solution of the following homogenous equation

$$
\left\{\begin{array}{l}
\partial_{z_{1}}\left(a_{1}\left(z_{1}\right) \partial_{z_{1}} u\right)+\partial_{z_{2}}\left(a_{2}\left(z_{1}\right) \partial_{z_{2}} u\right)-a_{3}\left(z_{1}\right)\left(u\left(0, z_{2}\right)+\kappa\right)=0 \text { in } E \\
\partial_{z_{1}} u\left(0, z_{2}\right)-a_{4}\left(u\left(0, z_{2}\right)+\kappa\right)=0 \\
\partial_{z_{1}} u\left(1, z_{2}\right)=\partial_{z_{2}} u\left(z_{1}, 0\right)=\partial_{z_{2}} u\left(z_{1}, 1\right)=u(0,0)=0
\end{array}\right.
$$

then $(u, \kappa) \equiv(0,0)$ holds true.

Proof. Due to $u \in H^{1}(E), u\left(0, z_{2}\right) \in L^{2}[0,1]$ holds true. Thus, it follows from the regularity theory of weak solutions to second order elliptic equations in cornered domains (see [1] and [11]) that $u \in C^{1, \sigma}(\bar{E}) \cap C^{\infty}(\bar{E} \backslash\{(0,0)\} \cup\{(1,0)\} \cup\{(0,1)\} \cup$ $\{(1,1)\})$ for some $0<\sigma<1$. Furthermore, it can be checked that the boundary conditions are compatible at the corner points in (4.3), thus $u \in C^{2, \sigma}(\bar{E})$.

Set $v(z)=\partial_{z_{2}} u(z)$. It follows from (4.3) that

$$
\left\{\begin{array}{l}
\partial_{z_{1}}\left(a_{1}\left(z_{1}\right) \partial_{z_{1}} v\right)+\partial_{z_{2}}\left(a_{2}\left(z_{1}\right) \partial_{z_{2}} v\right)-a_{3}\left(z_{1}\right) v\left(0, z_{2}\right)=0 \text { in } E \\
\partial_{z_{1}} v\left(0, z_{2}\right)-a_{4} v\left(0, z_{2}\right)=0 \\
\partial_{z_{1}} v\left(1, z_{2}\right)=v\left(z_{1}, 0\right)=v\left(z_{1}, 1\right)=0
\end{array}\right.
$$

Set $v_{n}\left(z_{1}\right)=\int_{0}^{1} v(z) \sin \left(n \pi z_{2}\right) d z_{2}(n=1,2, \cdots)$. Then, it follows from (4.4) that

$$
\left\{\begin{array}{l}
\left(a_{1}\left(z_{1}\right) v_{n}^{\prime}\left(z_{1}\right)\right)^{\prime}-a_{2}\left(z_{1}\right)(n \pi)^{2} v_{n}\left(z_{1}\right)-a_{3}\left(z_{1}\right) v_{n}(0)=0, \\
v_{n}^{\prime}(0)-a_{4} v_{n}(0)=v_{n}^{\prime}(1)=0 .
\end{array}\right.
$$

It follows from the Maximum Principle and Hopf's Lemma that $v_{n}\left(z_{1}\right) \equiv 0$ in (4.5). Since $\left\{\sin \left(n \pi z_{2}\right)\right\}_{n=1}^{\infty}$ forms a complete basis in $H_{0}^{1}[0,1]$, thus $v(z) \equiv 0$ holds true. This implies that $u(z) \equiv u\left(z_{1}\right)$. Hence, $u\left(z_{1}\right)$ solves the following problem

$$
\left\{\begin{array}{l}
\left(a_{1}\left(z_{1}\right) u^{\prime}\left(z_{1}\right)\right)^{\prime}-a_{3}\left(z_{1}\right)(u(0)+\kappa)=0 \quad \text { in } \quad(0,1) \\
u^{\prime}(0)-a_{4}(u(0)+\kappa)=u^{\prime}(1)=u(0)=0 .
\end{array}\right.
$$


From this, one obtains easily $\kappa=0$ and $u\left(z_{1}\right) \equiv 0$. Thus, Lemma 4.1 is proved.

Next, we show the regularity of weak solution $(u, \kappa)$ to $(4.2)$ under some appropriate assumptions on $f(z)$ and $g_{i}\left(z_{2}\right)(i=1,2)$ in $(4.2)$.

Lemma 4.2. If $f \in C^{1, \alpha}(\bar{E}), g_{i} \in C^{1, \alpha}[0,1](i=1,2)$ and

$$
\partial_{z_{2}} f\left(z_{1}, 0\right)=\partial_{z_{2}} f\left(z_{1}, 1\right)=0, g_{i}^{\prime}(0)=g_{i}^{\prime}(1)=0(i=1,2),
$$

and $u \in H^{1}(E)$ is a weak solution of (4.2), then $u \in C^{2, \alpha}(\bar{E})$ admits

$$
\|u\|_{C^{1, \alpha}(\bar{E})} \leq C\left(\|f\|_{C^{\alpha}(\bar{E})}+\left\|\left(g_{1}, g_{2}\right)\right\|_{C^{\alpha}[0,1]}+\|u\|_{H^{1}(E)}+|\kappa|\right)
$$

and

$$
\|u\|_{C^{2, \alpha}(\bar{E})} \leq C\left(\|f\|_{C^{1, \alpha}(\bar{E})}+\left\|\left(g_{1}, g_{2}\right)\right\|_{C^{1, \alpha}[0,1]}+\|u\|_{H^{1}(E)}+|\kappa|\right) .
$$

Proof. Set

$$
E_{0}=\left\{\left(z_{1}, z_{2}\right): 0<z_{1}<1,-1<z_{2}<2\right\}
$$

and introduce the notation

$$
\bar{w}(z)=\left\{\begin{array}{l}
w\left(z_{1}, 2-z_{2}\right), \text { for } 1<z_{2}<2 \\
w\left(z_{1}, z_{2}\right), \text { for } 0 \leq z_{2} \leq 1 \\
w\left(z_{1},-z_{2}\right), \text { for }-1<z_{2}<0
\end{array}\right.
$$

Then $\bar{u}(z)$ satisfies

$$
\left\{\begin{array}{l}
\partial_{z_{1}}\left(a_{1}\left(z_{1}\right) \partial_{z_{1}} \bar{u}\right)+\partial_{z_{2}}\left(a_{2}\left(z_{1}\right) \partial_{z_{2}} \bar{u}\right)=\partial_{z_{1}} \bar{f}+a_{3}\left(z_{3}\right)\left(\bar{u}\left(0, z_{2}\right)+\kappa\right) \text { in } E_{0} \\
\partial_{z_{1}} \bar{u}\left(0, z_{2}\right)-a_{4}\left(\bar{u}\left(0, z_{2}\right)+\kappa\right)=\bar{g}_{1}\left(z_{2}\right) \\
\partial_{z_{1}} \bar{u}\left(1, z_{2}\right)=\bar{g}_{2}\left(z_{2}\right), \quad \partial_{z_{2}} \bar{u}\left(z_{1},-1\right)=\partial_{z_{2}} \bar{u}\left(z_{1}, 2\right)=\bar{u}(0,0)=0
\end{array}\right.
$$

Using the $L^{\infty}$ norm estimate in [11] and the standard interior and the boundary estimates for second order linear elliptic equations in [7], one gets $\bar{u} \in C^{1, \alpha}(\bar{E})$ and

$$
\|\bar{u}\|_{C^{1, \alpha}(\bar{E})} \leq C\left(\|\bar{f}\|_{C^{\alpha}\left(\bar{E}_{0}\right)}+\left\|\left(\bar{g}_{1}, \bar{g}_{2}\right)\right\|_{C^{\alpha}[-1,2]}+\left\|\bar{u}\left(0, z_{2}\right)\right\|_{L^{2}[-1,2]}+|\kappa|\right) .
$$

From this, $\bar{u}\left(0, z_{2}\right) \in C^{1, \alpha}[-1,2]$. Thus one can apply the same argument for $(4.11)$ to show that $u \in C^{2, \alpha}(\bar{E})$ such that

$$
\|\bar{u}\|_{C^{2, \alpha}(\bar{E})} \leq C\left(\|\bar{f}\|_{C^{1, \alpha}\left(\bar{E}_{0}\right)}+\left\|\left(\bar{g}_{1}, \bar{g}_{2}\right)\right\|_{C^{1, \alpha}[-1,2]}+\left\|\bar{u}\left(0, z_{2}\right)\right\|_{L^{2}[-1,2]}+|\kappa|\right) .
$$

Combining (4.11)-(4.12) with the trace theorem yields (4.8)-(4.9), we complete the proof of Lemma 4.2 .

Finally, we prove the existence of a weak solution to (4.2).

Lemma 4.3. Under the assumptions in Lemma 4.2, (4.2) has a unique weak solution $(u(z), \kappa)$, such that $u(z) \in H^{1}(E)$ and the following estimate holds:

$$
\|u\|_{H^{1}(E)}+|\kappa| \leq C\left(\|f\|_{C^{\alpha}(\bar{E})}+\left\|\left(g_{1}, g_{2}\right)\right\|_{C^{1, \alpha}[0,1]}\right)
$$


Proof. First we split the equation (4.2) into the following two problems

$$
\left\{\begin{array}{l}
\partial_{z_{1}}\left(a_{1}\left(z_{1}\right) \partial_{z_{1}} u_{1}\right)+\partial_{z_{2}}\left(a_{2}\left(z_{1}\right) \partial_{z_{2}} u_{1}\right)-a_{3}\left(z_{1}\right) u_{1}\left(0, z_{2}\right)=\partial_{z_{1}} f \text { in } E \\
\partial_{z_{1}} u_{1}\left(0, z_{2}\right)-a_{4} u_{1}\left(0, z_{2}\right)=g_{1}\left(z_{2}\right), \quad \partial_{z_{1}} u_{1}\left(1, z_{2}\right)=g_{2}\left(z_{2}\right) \\
\partial_{z_{2}} u_{1}\left(z_{1}, 0\right)=\partial_{z_{2}} u_{1}\left(z_{1}, 1\right)=0
\end{array}\right.
$$

and

$$
\left\{\begin{array}{l}
\partial_{z_{1}}\left(a_{1}\left(z_{1}\right) \partial_{z_{1}} u_{2}\right)+\partial_{z_{2}}\left(a_{2}\left(z_{1}\right) \partial_{z_{2}} u_{2}\right)-a_{3}\left(z_{1}\right)\left(u_{2}\left(0, z_{2}\right)+\kappa\right)=0 \text { in } E \\
\partial_{z_{1}} u_{2}\left(0, z_{2}\right)-a_{4}\left(u_{2}\left(0, z_{2}\right)+\kappa\right)=0, \quad \partial_{z_{1}} u_{2}\left(1, z_{2}\right)=0 \\
\partial_{z_{2}} u_{2}\left(z_{1}, 0\right)=\partial_{z_{2}} u_{2}\left(z_{1}, 1\right)=0, \quad u_{2}(0,0)=-u_{1}(0,0)
\end{array}\right.
$$

It is noted that the uniqueness of $H^{1}(E)$ solution of (4.14) can be derived exactly as in Lemma 4.1. On the other hand, by a standard method (see [10] or [12]), one can establish the coerciveness and boundedness of the corresponding bilinear form to the following problem

$$
\left\{\begin{array}{l}
\partial_{z_{1}}\left(a_{1}\left(z_{1}\right) \partial_{z_{1}} u_{1}\right)+\partial_{z_{2}}\left(a_{2}\left(z_{1}\right) \partial_{z_{2}} u_{1}\right)-K u_{1}(z)-a_{3}\left(z_{1}\right) u_{1}\left(0, z_{2}\right)=\partial_{z_{1}} f \text { in } E, \\
\partial_{z_{1}} u_{1}\left(0, z_{2}\right)-a_{4} u_{1}\left(0, z_{2}\right)=g_{1}\left(z_{2}\right), \quad \partial_{z_{1}} u_{1}\left(1, z_{2}\right)=g_{2}\left(z_{2}\right), \\
\partial_{z_{2}} u_{1}\left(z_{1}, 0\right)=\partial_{z_{2}} u_{1}\left(z_{1}, 1\right)=0
\end{array}\right.
$$

with $K$ being a suitably large positive constant. Thus, by Lax-Milgram Theorem, we know that (4.16) is uniquely solvable in $H^{1}(E)$. Furthermore, by the Fredholm alternative theorem, (4.14) has a unique $H^{1}(E)$ solution which satisfies (4.13).

In addition, by the uniqueness of solution and a simple computation that the solution of (4.15) is

$$
u_{2}(z)=-u_{1}(0,0), \quad \kappa=u_{1}(0,0) .
$$

Thus, we complete the proof of Lemma 4.3.

Based on Lemma 4.1-4.3, we can now study the well-posedness of (3.11).

Proposition 4.4. When

$$
\lambda_{6}\left(z_{1}\right)+\frac{d}{d z_{1}}\left(\frac{\lambda_{3}\left(z_{1}\right) \lambda_{4}\left(z_{1}\right)}{\lambda_{2}\left(z_{1}\right)}\right)>0, \quad B_{1} \lambda_{2}(0) \lambda_{7}-\lambda_{3}(0)<0,
$$

then (3.11) has a unique solution $\left(\bar{W}_{2}, \bar{W}_{3} ; \bar{W}_{5}\left(-\theta_{0}\right)\right) \in C^{2, \alpha}\left(\bar{E}_{+}\right) \times \mathbb{R}$ satisfying

$$
\begin{aligned}
& \left\|\left(\bar{W}_{2}, \bar{W}_{3}\right)\right\|_{C^{l, \alpha}\left(\bar{E}_{+}\right)}+\left|\bar{W}_{5}\left(-\theta_{0}\right)\right| \\
\leq & C\left(\left\|\left(G_{1}, G_{2}\right)\right\|_{C^{l-1, \alpha}\left(\bar{E}_{+}\right)}+\left\|\left(G_{3}, \varepsilon P_{0}\right)\right\|_{C^{l, \alpha}\left[-\theta_{0}, \theta_{0}\right]}\right) \quad \text { for } l=1,2
\end{aligned}
$$

and

$$
\bar{W}_{2}\left(z_{1}, \pm \theta_{0}\right)=\partial_{z_{2}}^{2} \bar{W}_{2}\left(z_{1}, \pm \theta_{0}\right)=0, \quad \partial_{z_{2}} \bar{W}_{3}\left(z_{1}, \pm \theta_{0}\right)=0
$$

Proof. It is noted that the background solution is smooth, then the coefficients in (3.18) are also smooth. Due to (2.8), (3.7) and (4.18), it can be checked easily all the assumptions for Lemma 4.1-4.3 are satisfied for the problem (3.18), and hence, (3.18) has a unique solution $\left(\phi, \bar{W}_{5}\left(-\theta_{0}\right)\right) \in C^{2, \alpha}\left(\bar{E}_{+}\right) \times \mathbb{R}$ which satisfies for $l=0,1$ 
$(4.21)$

$$
\|\phi\|_{C^{1+l, \alpha}(\bar{E})}+\left|\bar{W}_{5}\left(-\theta_{0}\right)\right| \leq C\left(\left\|\left(G_{1}, G_{2}\right)\right\|_{C^{l, \alpha}\left(\bar{E}_{+}\right)}+\left\|\left(G_{3}, \varepsilon P_{0}\right)\right\|_{C^{1, \alpha}\left[-\theta_{0}, \theta_{0}\right]}\right) .
$$

By (3.13), (3.11) has a unique solution $\left(\bar{W}_{2}, \bar{W}_{3} ; \bar{W}_{5}\left(-\theta_{0}\right)\right) \in C^{1, \alpha} \times \mathbb{R}$ such that $(4.22)$

$\left\|\left(\bar{W}_{2}, \bar{W}_{3}\right)\right\|_{C^{\alpha}\left(\bar{E}_{+}\right)}+\left|\bar{W}_{5}\left(-\theta_{0}\right)\right| \leq C\left(\left\|\left(G_{1}, G_{2}\right)\right\|_{C^{\alpha}\left(\bar{E}_{+}\right)}+\left\|\left(G_{3}, \varepsilon P_{0}\right)\right\|_{C^{1, \alpha}\left[-\theta_{0}, \theta_{0}\right]}\right)$

and

$$
\bar{W}_{2}\left(z_{1}, \pm \theta_{0}\right)=0, \quad \partial_{z_{2}} \bar{W}_{3}\left(z_{1}, \pm \theta_{0}\right)=0 .
$$

Next, we estimate $\left\|\left(\bar{W}_{2}, \bar{W}_{3}\right)\right\|_{C^{k, \alpha}\left(\bar{E}_{+}\right)}$for $k=1,2$.

It is noted that (3.11) can be rewritten as

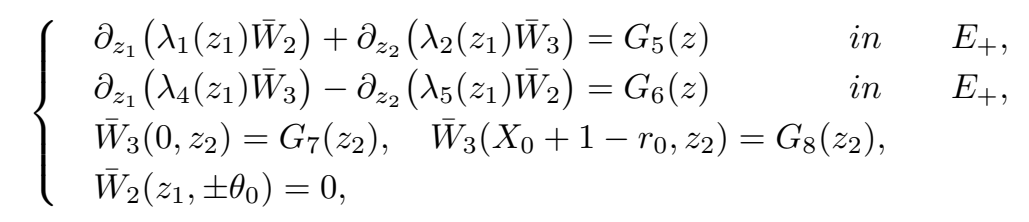

where

$$
\begin{aligned}
& G_{5}(z)=G_{1}(z)+\lambda_{3}\left(z_{1}\right) \bar{W}_{2}\left(0, z_{2}\right) \\
& G_{6}(z)=G_{2}(z)-\lambda_{6}\left(z_{1}\right)\left(\bar{W}_{5}\left(-\theta_{0}\right)+\lambda_{7} \int_{-\theta_{0}}^{z_{2}} \bar{W}_{2}(0, s) d s\right) \\
& G_{7}\left(z_{2}\right)=G_{3}\left(z_{2}\right)+B_{4}\left(\bar{W}_{5}\left(-\theta_{0}\right)+\lambda_{7} \int_{-\theta_{0}}^{z_{2}} \bar{W}_{2}(0, s) d s\right), G_{8}\left(z_{2}\right)=\varepsilon P_{0}\left(z_{2}\right)
\end{aligned}
$$

and

$$
G_{5}\left(z_{1}, \pm \theta_{0}\right)=0, \quad G_{6}^{\prime}\left(z_{1}, \pm \theta_{0}\right)=0, \quad G_{j}^{\prime}\left( \pm \theta_{0}\right)=0(j=7,8) .
$$

From (4.24), we can obtain

$$
\left\{\begin{array}{l}
\partial_{z_{1}}\left(\frac{\lambda_{1}\left(z_{1}\right)}{\lambda_{5}\left(z_{1}\right)} \partial_{z_{1}}\left(\lambda_{4}\left(z_{1}\right) \bar{W}_{3}\right)\right)+\partial_{z_{2}}^{2}\left(\lambda_{2}\left(z_{1}\right) \bar{W}_{3}\right)=\partial_{z_{1}}\left(\frac{\lambda_{1}\left(z_{1}\right)}{\lambda_{5}\left(z_{1}\right)} G_{6}(z)\right)+\partial_{z_{2}} G_{5}(z) i n E_{+}, \\
\bar{W}_{3}\left(0, z_{2}\right)=G_{7}\left(z_{2}\right), \quad \bar{W}_{3}\left(X_{0}+1-r_{0}, z_{2}\right)=G_{8}\left(z_{2}\right) \\
\partial_{z_{2}} \bar{W}_{3}\left(z_{1}, 0\right)=\partial_{z_{2}} \bar{W}_{3}\left(z_{1}, 1\right)=0 .
\end{array}\right.
$$

Note that (4.25) implies that the boundary conditions in (4.26) are compatible at the corners. So similar arguments as for Lemma 4.2 show that $\bar{W}_{3}$ admits the following estimates in $\bar{E}_{+}$for $l=1,2$

$$
\left\|\bar{W}_{3}\right\|_{C^{l, \alpha}} \leq C\left(\left\|\bar{W}_{2}\right\|_{C^{l-1, \alpha}}+\left|\bar{W}_{5}\left(-\theta_{0}\right)\right|+\left\|\left(G_{1}, G_{2}\right)\right\|_{C^{l-1}}+\left\|\left(G_{3}, \varepsilon P_{0}\right)\right\|_{C^{l, \alpha}}\right) .
$$

This, together with (4.24), yields in $\bar{E}_{+}$that for $l=1,2$

(4.28) $\left\|\bar{W}_{2}\right\|_{C^{l, \alpha}} \leq C\left(\left\|\bar{W}_{2}\right\|_{C^{l-1, \alpha}}+\left|\bar{W}_{5}\left(-\theta_{0}\right)\right|+\left\|\left(G_{1}, G_{2}\right)\right\|_{C^{l-1}}+\left\|\left(G_{3}, \varepsilon P_{0}\right)\right\|_{C^{l, \alpha}}\right)$.

Due to $\partial_{z_{2}} G_{6}\left(z_{1}, \pm \theta_{0}\right)=0$ and $\partial_{z_{2}} \bar{W}_{3}\left(z_{1}, \pm \theta_{0}\right)=0$, then it follows from the second equation in (4.24) that

$$
\partial_{z_{2}}^{2} \bar{W}_{2}\left(z_{1}, 0 \pm \theta_{0}\right)=0
$$

Thus, (4.19) can be derived from (4.27)-(4.28) together with (4.22). In addition, (4.20) has been proved in (4.23) and (4.29). Thus, we complete proof of Prop.4.4. 


\section{A priori estimates and proofs of Theorem 2.1 and Theorem 1.1.}

In this section, we establish some key a priori estimates on the linearized problems given in $\S 3$ to define a contractible mapping from $\Xi_{\delta}$ into $\Xi_{\delta}$ so that Theorem 2.1 and Theorem 1.1 can be shown. To this end, we first derive some a priori estimates on solutions to (3.3), (3.5), (3.18), and (3.19).

1. Solving the first order elliptic system (3.11) and estimating $\bar{W}_{2}, \bar{W}_{3}$ and $\bar{W}_{5}\left(-\theta_{0}\right)$.

As stated in the below of (3.18) of $\S 3$, if one can verify

$$
\lambda_{6}\left(z_{1}\right) \lambda_{7}+\frac{d}{d z_{1}}\left(\frac{\lambda_{3}\left(z_{1}\right) \lambda_{4}\left(z_{1}\right)}{\lambda_{2}\left(z_{1}\right)}\right)>0, \quad B_{4} \lambda_{7} \lambda_{2}(0)-\lambda_{3}(0)<0,
$$

then, due to (2.8) and (3.9)-(3.10), the conditions of Proposition 4.4 in $\S 4$ will be fulfilled. Thus, the solvability and the estimates on $\bar{W}_{2}, \bar{W}_{3}$ and $\bar{W}_{5}\left(-\theta_{0}\right)$ can be obtained by Proposition 4.4.

It follows from a direct computation bases on the background solution that

$$
\begin{aligned}
& \lambda_{6}\left(z_{1}\right) \lambda_{7}+\frac{d}{d z_{1}}\left(\frac{\lambda_{3}\left(z_{1}\right) \lambda_{4}\left(z_{1}\right)}{\lambda_{2}\left(z_{1}\right)}\right)=\lambda_{7} \lambda_{4}\left(z_{1}\right) B_{5} B_{2}\left(z_{1}\right)>0, \\
& B_{4} \lambda_{7} \lambda_{2}(0)-\lambda_{3}(0) \\
= & \frac{\lambda_{7} \lambda_{2}(0)\left(\hat{\rho}_{0}^{+} \hat{U}_{0}^{+}\right)\left(r_{0}\right)\left(\hat{U}_{0}^{+}\left(r_{0}\right)-U_{0}^{-}\left(r_{0}\right)\right)}{r_{0}\left(c^{2}\left(\hat{\rho}_{0}^{+}, S_{0}^{+}\right)-\left(\hat{U}_{0}^{+}\right)^{2}\right)\left(r_{0}\right)}\left((\gamma-1)\left(\hat{U}_{0}^{+}\left(r_{0}\right)\right)^{2}+c^{2}\left(\hat{\rho}_{0}^{+}, S_{0}^{+}\right)\right)<0 .
\end{aligned}
$$

Thus, (5.1) holds. Consequently, by Proposition 4.4 in $\S 4$, (3.11) has a unique solution $\left(\bar{W}_{2}, \bar{W}_{3}, \bar{W}_{5}\left(-\theta_{0}\right)\right)$ satisfying in $\bar{E}_{+}$

$$
\left\|\left(\bar{W}_{2}, \bar{W}_{3}\right)\right\|_{C^{2, \alpha}}+\left|\bar{W}_{5}\left(-\theta_{0}\right)\right| \leq C\left(\left\|\left(G_{1}, G_{2}\right)\right\|_{C^{1, \alpha}}+\left\|\left(G_{3}, \varepsilon P_{0}\right)\right\|_{C^{2, \alpha}}\right) \leq C\left(\varepsilon+\delta^{2}\right)
$$

and

$$
\bar{W}_{2}\left(z_{1}, \pm \theta_{0}\right)=\partial_{z_{2}}^{2} \bar{W}_{2}\left( \pm \theta_{0}\right)=0, \quad \partial_{z_{2}} \bar{W}_{3}\left(z_{1}, \pm \theta_{0}\right)=0
$$

2. Estimating $\bar{W}_{5}\left(z_{2}\right)$ in (3.3).

By the estimates in (5.2), the unique solution $\bar{W}_{5}\left(z_{2}\right)$ of (3.3) satisfies

$$
\left\|\bar{W}_{5}\right\|_{C^{3, \alpha}\left[-\theta_{0}, \theta_{0}\right]} \leq C\left(\left|\bar{W}_{5}\left(-\theta_{0}\right)\right|+\left\|\bar{W}_{2}\right\|_{C^{2, \alpha}\left(\bar{E}_{+}\right)}+\left\|F_{6}(z)\right\|_{C^{2, \alpha}\left(\bar{E}_{+}\right)}\right) \leq C\left(\varepsilon+\delta^{2}\right) .
$$

Furthermore, it follows from (3.4) and (5.3) that

$$
\bar{W}_{5}^{\prime}\left( \pm \theta_{0}\right)=\bar{W}_{5}^{(3)}\left( \pm \theta_{0}\right)=0 .
$$

3. Estimating $\bar{W}_{4}(z)$ in (3.5).

It follows from (3.6)-(3.7) and (5.4) that

$$
\left\|\bar{W}_{4}\right\|_{C^{2, \alpha}\left(\bar{E}_{+}\right)} \leq C\left(\left\|\bar{W}_{5}\right\|_{C^{2, \alpha}\left[-\theta_{0}, \theta_{0}\right]}+\left\|F_{7}(z)\right\|_{C^{2, \alpha}\left(\bar{E}_{+}\right)}\right) \leq C\left(\varepsilon+\delta^{2}\right) .
$$

Moreover, by use of (3.6)-(3.7) and (5.5), we arrive at

$$
\partial_{z_{2}} \bar{W}_{4}\left(z_{1}, \pm \theta_{0}\right)=0 \text {. }
$$

4. Estimating $\bar{W}_{1}(z)$ in (3.19). 
It follows from (3.19)-(3.20) and (5.2)-(5.7) that

$$
\left\|\bar{W}_{1}\right\|_{C^{2, \alpha}\left(\bar{E}_{+}\right)} \leq C\left(\left\|\left(\bar{W}_{3}, \bar{W}_{4}, F_{5}\right)\right\|_{C^{2, \alpha}\left(\bar{E}_{+}\right)}\right) \leq C\left(\varepsilon+\delta^{2}\right)
$$

and

$$
\partial_{z_{2}} \bar{W}_{1}\left(z_{1}, \pm \theta_{0}\right)=0 \text {. }
$$

We are now ready to show Theorem 2.1.

\section{Proof of Theorem 2.1.}

Based on the iteration scheme in $\S 3$ and the estimates (5.2)-(5.9), we can define a mapping $T$ as follows

$$
T(\hat{W})=\bar{W},
$$

where $\hat{W}=\left(\hat{W}_{1}, \hat{W}_{2}, \hat{W}_{3}, \hat{W}_{4}, \hat{W}_{5}\right)$ and $\bar{W}=\left(\bar{W}_{1}, \bar{W}_{2}, \bar{W}_{3}, \bar{W}_{4}, \bar{W}_{5}\right)$, moreover, $T$ is a continuous mapping from $\Xi_{\delta}$ into itself for properly chosen $\delta=O(1) \varepsilon>0$.

It remains to show that the mapping $T$ is contractible.

For any given two states $\hat{W}^{1}=\left(\hat{W}_{1}^{1}, \hat{W}_{2}^{1}, \hat{W}_{3}^{1}, \hat{W}_{4}^{1}, \hat{W}_{5}^{1}\right)$ and $\hat{W}^{2}=\left(\hat{W}_{1}^{2}, \hat{W}_{2}^{2}\right.$, $\left.\hat{W}_{3}^{2}, \hat{W}_{4}^{2}, \hat{W}_{5}^{2}\right)$ in $\Xi_{\delta}$ with the corresponding fluid variables $\left(\hat{U}_{11}, \hat{\omega}_{1}, \hat{P}_{1}, \hat{S}_{1}, \hat{\xi}_{1}\right)$ and $\left(\hat{U}_{12}, \hat{\omega}_{2}, \hat{P}_{2}, \hat{S}_{2}, \hat{\xi}_{2}\right)$ respectively, we set $T\left(\hat{W}^{1}\right)=\bar{W}^{1}$ and $T\left(\hat{W}^{2}\right)=\bar{W}^{2}$ with $\bar{W}^{i}=$ $\left(\bar{W}_{1}^{i}, \bar{W}_{2}^{i}, \bar{W}_{3}^{i}, \bar{W}_{4}^{i}, \bar{W}_{5}^{i}\right)$ for $i=1,2$.

Let

$\hat{Y}(z)=\left(\hat{Y}_{1}(z), \hat{Y}_{2}(z), \hat{Y}_{3}(z), \hat{Y}_{4}(z), \hat{Y}_{5}\left(z_{2}\right)\right), \bar{Y}(z)=\left(\bar{Y}_{1}(z), \bar{Y}_{2}(z), \bar{Y}_{3}(z), \bar{Y}_{4}(z), \bar{Y}_{5}\left(z_{2}\right)\right)$ with $\hat{Y}_{i}(z)=\hat{W}_{i}^{1}-\hat{W}_{i}^{2}$ and $\bar{Y}_{i}(z)=\bar{W}_{i}^{1}-\bar{W}_{i}^{2}(1 \leq i \leq 5)$.

In order to obtain the contractibility of $T$ in $\Xi_{\delta}$, we establish some estimates on $\bar{Y}_{i}$ for $1 \leq i \leq 5$, which will be provided by the following four steps.

\section{Step 1. The estimate of shock location deviation.}

It follows from (3.3) and a direct computation that

$$
\bar{Y}_{5}^{\prime}\left(z_{2}\right)=O(1) \bar{Y}_{2}\left(0, z_{2}\right)+O(\varepsilon) \hat{Y} .
$$

This implies

$$
\left\|\bar{Y}_{5}^{\prime}\right\|_{C^{1, \alpha}\left[-\theta_{0}, \theta_{0}\right]} \leq C\left\|\bar{Y}_{2}\right\|_{C^{1, \alpha}\left(\bar{E}_{+}\right)}+C \varepsilon\left(\sum_{i=1}^{4}\left\|\hat{Y}_{i}\right\|_{C^{1, \alpha}\left(\bar{E}_{+}\right)}+\left\|\hat{Y}_{5}\right\|_{C^{1, \alpha}\left[-\theta_{0}, \theta_{0}\right]}\right) .
$$

Step 2. The estimate of the entropy difference.

First, define the characteristics $z_{2}^{i}\left(s ; \beta_{i}\right)$ going through $\left(z_{1}, z_{2}\right)$ with $z_{2}^{i}\left(0 ; \beta_{i}\right)=\beta_{i}$ for $i=1,2$ as in $\S 3$, then a direct computation yields

$$
\begin{aligned}
& \left\|\beta_{1}-\beta_{2}\right\|_{C^{1, \alpha}\left(\bar{E}_{+}\right)} \leq C\left(\left\|\hat{Y}_{2}\right\|_{C^{1, \alpha}\left(\bar{E}_{+}\right)}+\varepsilon\left\|\hat{Y}_{5}\right\|_{C^{2, \alpha}\left[-\theta_{0}, \theta_{0}\right]}\right) \\
& \bar{Y}_{4}(z)=O(\varepsilon)\left(\beta_{1}-\beta_{2}\right)+O(1) \bar{Y}_{5}\left(\beta_{2}\right)+\sum_{i=1}^{4} O(\varepsilon) \hat{Y}_{i}\left(0, \beta_{2}\right)+B_{5} \int_{z_{2}}^{\beta_{2}(z)} \hat{Y}_{5}^{\prime}(s) d s
\end{aligned}
$$

Thus,

$$
\left\|\bar{Y}_{4}\right\|_{C^{1, \alpha}\left(\bar{E}_{+}\right)} \leq C\left(\left\|\bar{Y}_{5}\right\|_{C^{1, \alpha}\left[-\theta_{0}, \theta_{0}\right]}+\varepsilon \sum_{i=1}^{4}\left\|\hat{Y}_{i}\right\|_{C^{1, \alpha}\left(\bar{E}_{+}\right)}+\varepsilon\left\|\hat{Y}_{5}\right\|_{C^{2, \alpha}\left[-\theta_{0}, \theta_{0}\right]}\right) .
$$

Step 3. The estimates of $\bar{Y}_{2}(z), \bar{Y}_{3}(z)$ and $\bar{Y}_{5}\left(-\theta_{0}\right)$. It is noted that $\bar{Y}_{2}, \bar{Y}_{3}$ and $\bar{Y}_{5}\left(-\theta_{0}\right)$ satisfy 
(5.16)

$$
\left\{\begin{array}{l}
\partial_{z_{1}}\left(\lambda_{1}\left(z_{1}\right) \bar{Y}_{2}\right)+\partial_{z_{2}}\left(\lambda_{2}\left(z_{1}\right) \bar{Y}_{3}\right)-\lambda_{3}\left(z_{1}\right) \bar{Y}_{2}\left(0, z_{2}\right)=\sum_{i=1}^{5}\left(O(\varepsilon) \hat{Y}_{i}+O(\varepsilon) \nabla \hat{Y}_{i}\right), \\
\left.\partial_{z_{1}}\left(\lambda_{4}\left(z_{1}\right) \bar{Y}_{3}\right)-\partial_{z_{2}}\left(\lambda_{5}\left(z_{1}\right) \bar{Y}_{2}\right)+\lambda_{6}\left(\bar{Y}_{5}\left(-\theta_{0}\right)+\lambda_{7} \int_{-\theta_{0}}^{z_{2}} \bar{Y}_{2}(0, s) d s\right) Y_{i}+O(\varepsilon) \nabla \hat{Y}_{i}\right) \\
=\sum_{i=1}^{5}\left(O(\varepsilon) \hat{+} O(\varepsilon)\left(\beta_{1}-\beta_{2}\right)+O(1) \int_{z_{2}}^{\beta_{2}(z)} \hat{Y}_{5}^{\prime}(s) d s,\right. \\
\bar{Y}_{3}\left(0, z_{2}\right)=B_{1}\left(\bar{Y}_{5}\left(-\theta_{0}\right)+\lambda_{7} \int_{-\theta_{0}}^{z_{2}} \bar{Y}_{2}(0, s) d s\right)+\sum_{i=1}^{5} O(\varepsilon) \hat{Y}_{i}, \\
\bar{Y}_{3}\left(X_{0}+1-r_{0}, z_{2}\right)=\bar{Y}_{2}\left(z_{1}, \pm \theta_{0}\right)=0 .
\end{array}\right.
$$

Thus, it follows from (4.19) with $l=1$ that

$$
\left\|\bar{Y}_{2}\right\|_{C^{1, \alpha}\left(\bar{E}_{+}\right)}+\left\|\bar{Y}_{3}\right\|_{C^{1, \alpha}\left(\bar{E}_{+}\right)}+\left|\bar{Y}_{5}\left(-\theta_{0}\right)\right| \leq C \varepsilon\left(\sum_{i=1}^{4}\left\|\hat{Y}_{i}\right\|_{C^{1, \alpha}\left(\bar{E}_{+}\right)}+\left\|\hat{Y}_{5}\right\|_{C^{2, \alpha}\left[-\theta_{0}, \theta_{0}\right]}\right) .
$$

Step 4. The estimate of $\bar{Y}_{1}(z)$.

It follows from (3.19) that

$$
\bar{Y}_{1}=O(1) \bar{Y}_{3}+O(1) \bar{Y}_{4}+O(\varepsilon)\left(\hat{Y}_{1}, \hat{Y}_{2}, \hat{Y}_{3}, \hat{Y}_{4}\right)
$$

Thus,

$$
\left\|\bar{Y}_{1}\right\|_{C^{1, \alpha}\left(\bar{E}_{+}\right)} \leq C\left(\left\|\bar{Y}_{3}\right\|_{C^{1, \alpha}\left(\bar{E}_{+}\right)}+\left\|\bar{Y}_{4}\right\|_{C^{1, \alpha}\left(\bar{E}_{+}\right)}+\varepsilon \sum_{i=1}^{4}\left\|\hat{Y}_{i}\right\|_{C^{1, \alpha}\left(\bar{E}_{+}\right)}\right) .
$$

Collecting all the estimates in Step 1-Step 4 above shows that

$$
\sum_{i=1}^{4}\left\|\bar{Y}_{i}\right\|_{C^{1, \alpha}\left(\bar{E}_{+}\right)}+\left\|\bar{Y}_{5}\right\|_{C^{2, \alpha}\left[-\theta_{0}, \theta_{0}\right]} \leq C \varepsilon\left(\sum_{j=1}^{4}\left\|\hat{Y}_{j}\right\|_{C^{1, \alpha}\left(\bar{E}_{+}\right)}+\left\|\hat{Y}_{5}\right\|_{C^{2, \alpha}\left[-\theta_{0}, \theta_{0}\right]}\right) .
$$

Thus, for suitably small $\varepsilon$, (5.20) implies that $T$ is contractible in $\left(C^{1, \alpha}\left(E_{+}\right)\right)^{4} \times$ $C^{2, \alpha}\left[-\theta_{0}, \theta_{0}\right]$. Therefore, there exists a unique solution $W$ in $\Xi_{\delta}$ which solves (2.20)(2.23). Furthermore, by the definition of $\Xi_{\delta}$, we know that $W$ satisfies (2.24). Hence, we complete the proof of Theorem 2.1.

Finally, we prove Theorem 1.1.

Proof of Theorem 1.1. By Theorem 2.1, there exists a unique solution $\left(U_{1}(z)\right.$, $\left.\omega(z), P(z), S(z) ; \xi\left(z_{2}\right)\right)$ to (2.16)-(2.18) and (2.13), and further $\left(U_{1}(r, \theta), \omega(r, \theta)\right.$, $P(r, \theta), S(r, \theta) ; \xi(\theta))$ solves (2.10)-(2.13). Moreover, in terms of (2.1), one obtains a solution $\left(u_{1}(x), u_{2}(x), P(x), S(x) ; \eta\left(x_{2}\right)\right)$ to (1.1) with (1.2)-(1.5) and admits

$$
\left\|\eta\left(x_{2}\right)-\sqrt{r_{0}^{2}-x_{2}^{2}}\right\|_{C^{3, \alpha}\left[x_{2}^{1}, x_{2}^{2}\right]} \leq C\left\|\xi-r_{0}\right\|_{C^{3, \alpha}\left[-\theta_{0}, \theta_{0}\right]} \leq C \varepsilon
$$

here $\left(x_{1}^{i}, x_{2}^{i}\right)$ is the intersection points of $x_{1}=\eta\left(x_{2}\right)$ with $x_{2}=(-1)^{i} x_{1} \tan \theta_{0}$, and

$$
\begin{aligned}
& \left\|\left(u_{1}(x), u_{2}(x), P(x), S(x)\right)-\left(\hat{U}_{0}^{+}(r) \frac{x_{1}}{r}, \hat{U}_{0}^{+}(r) \frac{x_{2}}{r}, \hat{P}_{0}^{+}(r), S_{0}^{+}\right)\right\|_{C^{2, \alpha}\left(\bar{\Omega}_{+}\right)} \\
\leq & C\left(\left\|\left(U_{1}(z), \omega(z), P(z), S(z)\right)-\left(\tilde{U}_{0}^{+}\left(z_{1}\right), 0, \tilde{P}_{0}^{+}\left(z_{1}\right), S_{0}^{+}\right)\right\|_{C^{2, \alpha}}+\left\|\xi-r_{0}\right\|_{C^{2, \alpha}}\right) \\
\leq & C\left(\left\|\left(U_{1}(z), \omega(z), P(z), S(z)\right)-\left(\tilde{U}_{0}^{+}\left(z_{1}\right), 0, \tilde{P}_{0}^{+}\left(z_{1}\right), S_{0}^{+}\right)\right\|_{C^{2, \alpha}}+\left\|\xi-r_{0}\right\|_{C^{2, \alpha}}\right) \\
\leq & C \varepsilon
\end{aligned}
$$

Thus, the proof of Theorem 1.1 is completed. 


\section{Acknowledgements}

This research is supported part by National Basic Research Program of China (No. 2006CB805902), NSFC (No. 10571082), Zheng Ge Ru Foundation, Hong Kong RGC Earmarked Research Grants CUHK4028/04P, CUHK4040/06P, CUHK 4042/08P, and the RGC Central Allocation Grant CA05/06.SC01.

\section{References}

[1] A. Azzam, Smoothness properties of mixed boundary value problems for elliptic equations in sectionally smooth n-dimensional domains, Ann.Polon.Math. 40 (1981) 81-93.

[2] S. Canic, B. Keyfitz, and G. Lieberman, A proof of existence of perturbed steady transonic shocks via a free boundary problem.

[3] G. Chen, J. Chen, and K. Song, Transonic nozzle flows and free boundary problems for the full Euler equations, J.Differential Equations 229 (2006), no. 1, 92-120.

[4] S. Chen, Transonic shocks in 3-D compressible flow passing a duct with a general section for Euler systems, Trans. Amer. Math. Soc. 360 (2008), no. 10, 5265-5289.

[5] R. Courant and K. Friedrichs, Supersonic flow and shock waves, Interscience Publishers Inc., New York (1948).

[6] P. Embid, J. Goodman, and A. Majda, Multiple steady states for 1-D transonic flow, SIAM J. Sci. Statist. Comput. 5 (1984), no. 1, 21-41.

[7] D. Gilbarg and N. Tudinger, Elliptic partial differential equations of second order, Vol. 224 of Grundlehren der Mathematischen Wissenschaften, Springer, Berlin-New York, second edition edition (1983).

[8] F. John, Nonlinear wave equations, formation of singularities, Vol. 2 of University Lecture Series, American Mathematical Society, Providence, RI (1990).

[9] J. Li, Z. Xin, and H. Yin, On transonic shocks in a nozzle with variable end pressures, Preprint (2007)

[10] T. Li, S. Zheng, Y. Tan, and W. Shen, Boundary value problems with equivalued surface and resistivity well-logging, Vol. 382 of Pitman Research Notes in Mathematics Series, Longman, Harlow (1998).

[11] G. Lieberman, Mixed boundary value problems for elliptic and parabolic differential equation of second order, J.Math.Anal.Appl. 113 (1986), no. 2, 422-440.

[12] L. Liu and H. Yuan, Stability of cylindrical transonic shocks for the two-dimensional steady compressible Euler system, J. Hyperbolic Differ. Equ. 5 (2008), no. 2, 347-379.

[13] T. Liu, Transonic gas flow in a duct of varying area, Arch. Rational Mech. Anal. 80 (1982), no. $1,1-18$.

[14] C. Morawetz, Potential theory for regular and Mach reflection of a shock at a wedge, Comm. Pure Appl. Math. 47 (1994) 593-624.

[15] Z. Xin, W. Yan, and H. Yin, Transonic shock problem for the Euler system in a nozzle, Arch.Rat.Mech.Anal. (2008(in press))

[16] Z. Xin and H. Yin, Transonic shock in a curved nozzle, 2-D and 3-D complete Euler systems, J. D. E. 245 (2008), no. 4, 1014-1085.

The Institute of Mathematical Sciences, CUhK, Hong Kong,

E-mail address: lijun@nju.edu.cn

The Institute of Mathematical Sciences, CUHK, Hong Kong,

E-mail address: zpxin@ims.cuhk.edu.hk

Department of Mathematics \& IMS, Nanjing University, P. R. China,

E-mail address: huicheng@nju.edu.cn 